\title{
Mellin Transform Method for the Valuation of the American Power Put Option with Non-Dividend and Dividend Yields
}

\author{
Sunday Emmanuel Fadugba, Chuma Raphael Nwozo \\ ${ }^{1}$ Department of Mathematical Sciences, Ekiti State University, Ado Ekiti, Nigeria \\ ${ }^{2}$ Department of Mathematics, University of Ibadan, Oyo State, Nigeria \\ Email: emmasfad2006@yahoo.com, crnwozo@yahoo.com
}

Received 25 May 2015; accepted 4 July 2015; published 10 July 2015

Copyright (C) 2015 by authors and Scientific Research Publishing Inc.

This work is licensed under the Creative Commons Attribution International License (CC BY). http://creativecommons.org/licenses/by/4.0/

(c) (i) Open Access

\begin{abstract}
In this paper we present the Mellin transform method for the valuation of the American power put option with non-dividend and dividend yields, respectively. We use the Mellin transform method to derive the integral representations for the price and the free boundary of the American power put option. We also extend our results to derive the free boundary and the fundamental analytic valuation formula for perpetual American power put option which has no expiry date. Numerical experiments have shown that the Mellin transform method is a better alternative technique compared to the binomial model (BSM), recursive method (RM) and finite difference method (FDM) for the valuation of the American power put option. In general, the Mellin transform method is accurate, flexible and produces accurate prices for the optimal exercise boundary of the American power put option for a wide range of parameters. Hence the Mellin transform method is mutually consistent and agrees with the values of the analytic option valuation formula called the "BlackScholes model".
\end{abstract}

\section{Keywords}

American Power Option, Dividend Yield, Mellin Transform Method, Non-Dividend Yield, Perpetual Power Put Option

\section{Introduction}

Option valuation has been studied extensively in the last three decades. Many problems in financial mathematics entail the computation of a particular integral. In many cases these integrals can be valued analytically and in 
some cases they can be computed using a partial differential equation, or valued using numerical integration.

Power option is defined as a contingent claim on the product of powers of several underlying assets. The holder has either the right, but not the obligation to buy, as in the case of the power call option, or the possibility to sell, as in the case of the power put option, an asset for a certain price at a prescribed date in the future. The difference between the American and the European power options is that the European power option can only be exercised at the maturity or expiry date while the American power option can be exercised by its holder at any time before the expiry date. This early exercise feature makes the valuation of the American power option mathematically challenging and therefore, creats a great field of research.

A perpetual American power option is an option that has no expiry date. In other words, this type of power option never expires. In a special case of a plain vanilla perpetual option, a closed form solution for the free boundary and price of the American put was derived by [1].

Mellin transforms in option theory were introduced by [2], [3] extended the results obtained in [2] and showed how the Mellin transform approach could be used to derive the valuation formula for the perpetual American put options on dividend-paying stocks. [4] considered the Mellin transform method for the valuation of some vanilla power options with non-dividend yield. They derived the fundamental valuation formula known as the Black-Scholes model using the convolution property of the Mellin transform method. The analytical valuation of the American options was considered by [5]. An alternative approach to the valuation of American options and applications was considered by [6].

For the mathematical background of the Mellin transform method in derivatives valuation see [7]-[15], just to mention few. In this paper, we focus on the Mellin transform method for the valuation of the American power put option with non-dividend and dividend yields, respectively, and its extension to power option which has no expiry date, i.e. "perpetual American power put option". The rest of the paper is structured as follows: in Section 2, we present American power options and the payoffs for power call and put options. Section 3 presents the Mellin transform method for the valuation of the American power put option. Section 4 considers the extension of the Mellin transform method to the valuation of the perpetual American power put option. In Section 5, we present some numerical experiments. Section 6 concludes the paper.

\section{American Power Options}

The power options can be seen as a class of options in which the payoff at expiry is related to the $n^{\text {th }}$ power of the underlying price of the asset. American power options are options that can be exercised before or at the expiry date with non-linear payoff. The American power option comes in two forms, namely, the American power call option and the American power put option. The American power call option is an option with nonlinear payoff given by the difference between the price of the underlying asset at maturity raised to a strictly positive power and the exercise price. The American power put option is an option with non-linear payoff given by the difference between the exercise price and price of the underlying asset at maturity raised to a strictly positive power. For an American power option on the underlying price of the asset $S_{T}^{n}$ with exercise price $K$ and time to expiry $T$, we have the payoffs for the American power call and put options as

$$
A_{c}^{n}\left(S_{T}^{n}, t\right)=\left(S_{T}^{n}-K\right)^{+}=\max \left(S_{T}^{n}-K, 0\right)
$$

and

$$
A_{p}^{n}\left(S_{T}^{n}, t\right)=\left(K-S_{T}^{n}\right)^{+}=\max \left(K-S_{T}^{n}, 0\right)
$$

respectively.

\section{Remark 1}

- For $n=1$, the payoffs for American power call and put options in (1) and (2) become the payoffs for plain American call and put options, i.e.

$$
A_{c}\left(S_{T}, t\right)=\left(S_{T}-K\right)^{+}=\max \left(S_{T}-K, 0\right)
$$

and

$$
A_{p}\left(S_{T}, t\right)=\left(K-S_{T}\right)^{+}=\max \left(K-S_{T}, 0\right)
$$

respectively. 


\section{The Mellin Transform Method for the Valuation of the American Power Put Option}

There are many methods for the valuation of the American power option leading to different but equivalent mathematical formulations. We consider the derivation of the integral representation for the price of the American power put option and the integral equation to determine the free boundary of the American power put option via the Mellin transform method for the case of both non-dividend and dividend yields.

\subsection{American Power Put Option with Non-Dividend Yield}

Consider the non-homogeneous Black-Scholes partial differential equation for the American power put option with non-dividend yield given by

$$
\begin{aligned}
& \frac{\partial A_{p}^{n}\left(S_{t}^{n}, t\right)}{\partial t}+n\left(\frac{1}{2} \sigma^{2}(n-1)+r\right) S_{t}^{n} \frac{\partial A_{p}^{n}\left(S_{t}^{n}, t\right)}{\partial S_{t}^{n}} \\
& +\frac{1}{2}\left(\sigma n S_{t}^{n}\right)^{2} \frac{\partial^{2} A_{p}^{n}\left(S_{t}^{n}, t\right)}{\partial\left(S_{t}^{n}\right)^{2}}-r A_{p}^{n}\left(S_{t}^{n}, t\right)=f\left(S_{t}^{n}, t\right)
\end{aligned}
$$

where the early exercise function $f$ defined on $(0, \infty) \times(0, T)$ is given by

$$
f\left(S_{t}^{n}, t\right)= \begin{cases}-r K, & \text { if } 0<S_{t}^{n} \leq \hat{S}_{t}^{n} \\ 0, & \text { if } S_{t}^{n}>\hat{S}_{t}^{n}\end{cases}
$$

The final time condition given by $A_{p}\left(S_{t}^{n}, T\right)=\phi\left(S_{t}^{n}\right)=\max \left(K-S_{t}^{n}, 0\right)=\left(K-S_{t}^{n}\right)^{+}$on $[0, \infty)$ is called the high contact condition. The other boundary conditions are given by

$$
\begin{aligned}
& \lim _{S_{t}^{n} \rightarrow \infty} A_{p}^{n}\left(S_{t}^{n}, t\right)=0 \text { on }[0, T) \\
& \lim _{S_{t}^{n} \rightarrow 0} A_{p}^{n}\left(S_{t}^{n}, t\right)=K \text { on }[0, T)
\end{aligned}
$$

The free boundary $\hat{S}_{t}^{n}$ is determined by the smooth pasting conditions given by

$$
A_{p}\left(\hat{S}_{t}^{n}, t\right)=K-\hat{S}_{t}^{n}
$$

and

$$
\frac{\partial A_{p}\left(\hat{S}_{t}^{n}, t\right)}{\partial \hat{S}_{t}^{n}}=-1
$$

Applying the Mellin transform to (5), we have that

$$
\frac{\partial \tilde{A}_{p}^{n}(\omega, t)}{\partial t}+\frac{n^{2} \sigma^{2}}{2}\left(\omega^{2}+\omega\left(1-\frac{n-1}{n}-\frac{2 r}{n \sigma^{2}}\right)-\frac{2 r}{n^{2} \sigma^{2}}\right) \tilde{A}_{p}^{n}(\omega, t)=\tilde{f}(\omega, t)
$$

Setting $\alpha_{1}=\left(1-\frac{n-1}{n}-\frac{2 r}{n \sigma^{2}}\right)$ and $\alpha_{2}=\frac{2 r}{n^{2} \sigma^{2}}$. Then (11) yields

$$
\frac{\partial \tilde{A}_{p}^{n}(\omega, t)}{\partial t}+\frac{n^{2} \sigma^{2}}{2}\left(\omega^{2}+\omega \alpha_{1}-\alpha_{2}\right) \tilde{A}_{p}^{n}(\omega, t)=\tilde{f}(\omega, t)
$$

The Mellin transform of the early exercise function in (12) is obtained as

$$
\tilde{f}(\omega, t)=\int_{0}^{\infty} f\left(S_{t}^{n}, t\right)\left(S_{t}^{n}\right)^{\omega-1} \mathrm{~d} S_{t}^{n}=\int_{0}^{\hat{S}_{t}^{n}}-r K\left(S_{t}^{n}\right)^{\omega-1} \mathrm{~d} S_{t}^{n}=\frac{-r K\left(\hat{S}_{t}^{n}\right)^{\omega}}{\omega}
$$

Solving further, we have the particular solution of (12) as 


$$
\tilde{A}_{p}^{n}(\omega, t)_{(p . s o l)}=\int_{t}^{T} \frac{r K\left(\hat{S}_{t}^{n}\right)^{\omega}}{\omega} \mathrm{e}^{\frac{1}{2} n^{2} \sigma^{2}\left(\omega^{2}+\alpha_{1} \omega-\alpha_{2}\right)(y-t)} \mathrm{d} y
$$

The complementary solution to the left hand side of (12) is obtained as

$$
\tilde{A}_{p}^{n}(\omega, t)_{\text {comp.sol }}=c(\omega) \mathrm{e}^{-\frac{1}{2} n^{2} \sigma^{2}\left(\omega^{2}+\alpha_{1} \omega-\alpha_{2}\right) t}
$$

where $c(\omega)$ is the integration constant obtained as

$$
c(\omega)=\tilde{\phi}(\omega, t) \mathrm{e}^{\frac{1}{2} n^{2} \sigma^{2}\left(\omega^{2}+\alpha_{1} \omega-\alpha_{2}\right) T}
$$

$\tilde{\phi}(\omega, t)$ is the Mellin transform of the final time condition and is given by

$$
\tilde{\phi}(\omega, t)=\int_{0}^{\infty}\left(K-S_{T}^{n}\right)^{+}\left(S_{T}^{n}\right)^{\omega-1}=\int_{0}^{K}\left(K-S_{T}^{n}\right)\left(S_{T}^{n}\right)^{\omega-1}=\frac{K^{\omega+1}}{\omega(\omega+1)}
$$

Using (16) and (17) in (15) we have that

$$
\tilde{A}_{p}^{n}(\omega, t)_{\text {comp.sol }}=\frac{K^{\omega+1}}{\omega(\omega+1)} \mathrm{e}^{-\frac{1}{2} n^{2} \sigma^{2}\left(\omega^{2}+\alpha_{1} \omega-\alpha_{2}\right)(T-t)}
$$

Hence the general solution to (12) is given by

$$
\begin{aligned}
\tilde{A}_{p}^{n}(\omega, t)= & \tilde{A}_{p}^{n}(\omega, t)_{\text {comp.sol }}+\tilde{A}_{p}^{n}(\omega, t)_{(p . s o l)}=\frac{K^{\omega+1}}{\omega(\omega+1)} \mathrm{e}^{-\frac{1}{2} n^{2} \sigma^{2}\left(\omega^{2}+\alpha_{1} \omega-\alpha_{2}\right)(T-t)} \\
& +\int_{t}^{T} \frac{r K\left(\hat{S}_{y}^{n}\right)^{\omega}}{\omega} \mathrm{e}^{\frac{1}{2} n^{2} \sigma^{2}\left(\omega^{2}+\alpha_{1} \omega-\alpha_{2}\right)(y-t)} \mathrm{d} y
\end{aligned}
$$

The Mellin inversion of (19) is obtained as

$$
\begin{aligned}
A_{p}^{n}\left(S_{t}^{n}, t\right)= & \frac{1}{2 \pi i} \int_{c-i \infty}^{c+i \infty} \frac{K^{\omega+1}}{\omega(\omega+1)} \mathrm{e}^{-\frac{1}{2} n^{2} \sigma^{2}\left(\omega^{2}+\alpha_{1} \omega-\alpha_{2}\right)(T-t)}\left(S_{t}^{n}\right)^{-\omega} \mathrm{d} \omega \\
& +\frac{r K}{2 \pi i} \int_{c-i \infty}^{c+i \infty}\left(S_{t}^{n}\right)^{-\omega} \int_{t}^{T} \frac{\left(\hat{S}_{y}^{n}\right)^{\omega}}{\omega} \mathrm{e}^{\frac{1}{2} n^{2} \sigma^{2}\left(\omega^{2}+\alpha_{1} \omega-\alpha_{2}\right)(y-t)} \mathrm{d} y \mathrm{~d} \omega
\end{aligned}
$$

where $\left(S_{t}^{n}, t\right) \in\{(0, \infty) \times[0, T)\}, c \in(0, \infty)$ and $\{\omega \in C \mid 0<\operatorname{Re}(\omega)<\infty\}$.

\section{Remark 2}

- The first term in (20) is the integral representation for the price of the European power put option (stems from the minimum guaranteed payoff of the American power put) which pays no dividend yield (see [4]). The second term in (20) is called the early exercise premium (the value attributable to the right of exercising the option early) for the American power put option with non dividend yield denoted by $e_{p}^{n}\left(S_{t}^{n}, t\right)$. Therefore (20) becomes

$$
A_{p}^{n}\left(S_{t}^{n}, t\right)=E_{p}^{n}\left(S_{t}^{n}, t\right)+e_{p}^{n}\left(S_{t}^{n}, t\right)
$$

where

$$
\begin{aligned}
& E_{p}^{n}\left(S_{t}^{n}, t\right)=\frac{1}{2 \pi i} \int_{c-i \infty}^{c+i \infty} \frac{K^{\omega+1}}{\omega(\omega+1)} \mathrm{e}^{-\frac{1}{2} n^{2} \sigma^{2}\left(\omega^{2}+\alpha_{1} \omega-\alpha_{2}\right)(T-t)}\left(S_{t}^{n}\right)^{-\omega} \mathrm{d} \omega \\
& e_{p}^{n}\left(S_{t}^{n}, t\right)=\frac{r K}{2 \pi i} \int_{c-i \infty}^{c+i \infty}\left(S_{t}^{n}\right)^{-\omega} \int_{t}^{T} \frac{\left(\hat{S}_{y}^{n}\right)^{\omega}}{\omega} \mathrm{e}^{\frac{1}{2} n^{2} \sigma^{2}\left(\omega^{2}+\alpha_{1} \omega-\alpha_{2}\right)(y-t)} \mathrm{d} y \mathrm{~d} \omega
\end{aligned}
$$

- Setting $S_{t}^{n}=\hat{S}_{t}^{n}$ in (21) and using the smooth pasting conditions given by (9) and (10), we have the integral representation for the free boundary of the American power put option with non-dividend yield as 


$$
\hat{S}_{t}^{n}=K-E_{p}^{n}\left(\hat{S}_{t}^{n}, t\right)-\frac{r K}{2 \pi i} \int_{c-i \omega}^{c+i \omega}\left(\hat{S}_{t}^{n}\right)^{-\omega} \int_{t}^{T} \frac{\left(\hat{S}_{y}^{n}\right)^{\omega}}{\omega} \mathrm{e}^{\frac{1}{2} n^{2} \sigma^{2}\left(\omega^{2}+\alpha_{1} \omega-\alpha_{2}\right)(y-t)} \mathrm{d} y \mathrm{~d} \omega
$$

where

$$
E_{p}^{n}\left(\hat{S}_{t}^{n}, t\right)=\frac{1}{2 \pi i} \int_{c-i \infty}^{c+i \infty} \frac{K^{\omega+1}}{\omega(\omega+1)} \mathrm{e}^{-\frac{1}{2} n^{2} \sigma^{2}\left(\omega^{2}+\alpha_{1} \omega-\alpha_{2}\right)(T-t)}\left(\hat{S}_{t}^{n}\right)^{-\omega} \mathrm{d} \omega
$$

We formalized the properties highlighted in Remark $\mathbf{2}$ in the following results.

Theorem 1 The American power put option $A_{p}^{n}\left(S_{t}^{n}, t\right)$ which pays no dividend yield satisfies the decomposition

$$
A_{p}^{n}\left(S_{t}^{n}, t\right)=E_{p}^{n}\left(S_{t}^{n}, t\right)+\frac{r K}{2 \pi i} \int_{c-i \infty}^{c+i \infty}\left(S_{t}^{n}\right)^{-\omega} \int_{t}^{T} \frac{\left(\hat{S}_{y}^{n}\right)^{\omega}}{\omega} \mathrm{e}^{\frac{1}{2} n^{2} \sigma^{2}\left(\omega^{2}+\alpha_{1} \omega-\alpha_{2}\right)(y-t)} \mathrm{d} y \mathrm{~d} \omega
$$

where $\alpha_{1}=\left(1-\frac{n-1}{n}-\frac{2 r}{n \sigma^{2}}\right)$ and $\alpha_{2}=\frac{2 r}{n^{2} \sigma^{2}},\left(S_{t}^{n}, t\right) \in\{(0, \infty) \times[0, T)\}, c \in(0, \infty)$ and $\{\omega \in C \mid 0<\operatorname{Re}(\omega)<\infty\}$.

Theorem 2 Using the smooth pasting conditions given by $A_{p}\left(\hat{S}_{t}^{n}, t\right)=K-\hat{S}_{t}^{n}$ and $\frac{\partial A_{p}\left(\hat{S}_{t}^{n}, t\right)}{\partial \hat{S}_{t}^{n}}=-1$, the free boundary formulation of the American power put option with non-dividend yield is given by

$$
\hat{S}_{t}^{n}=K-E_{p}^{n}\left(\hat{S}_{t}^{n}, t\right)-\frac{r K}{2 \pi i} \int_{c-i \omega}^{c+i \omega}\left(\hat{S}_{t}^{n}\right)^{-\omega} \int_{t}^{T} \frac{\left(\hat{S}_{y}^{n}\right)^{\omega}}{\omega} \mathrm{e}^{\frac{1}{2} n^{2} \sigma^{2}\left(\omega^{2}+\alpha_{1} \omega-\alpha_{2}\right)(y-t)} \mathrm{d} y \mathrm{~d} \omega
$$

\subsection{American Power Put Option with Dividend Yield}

The derivation of the integral representation for the price of the American power put option which pays dividend yield using the Mellin transform method is given in the following result.

Theorem 3 Let $S_{t}^{n}$ be the price of the underlying asset, $K$ be the strike price, $r$ be the risk interest rate, $q$ be the dividend yield and $T$ be the time to maturity. Assume $S_{t}^{n}$ yields dividend, then the integral representation for the price of the American power put option $A_{p}^{n}\left(S_{t}^{n}, t\right)$ is given by

$$
\begin{aligned}
A_{p}^{n}\left(S_{t}^{n}, t\right)= & \frac{1}{2 \pi i} \int_{c-i \infty}^{c+i \infty} \frac{K^{\omega+1}}{\omega(\omega+1)} \mathrm{e}^{-\frac{1}{2} n^{2} \sigma^{2}\left(\omega^{2}+\alpha_{1}^{*} \omega-\alpha_{2}\right)(T-t)}\left(S_{t}^{n}\right)^{-\omega} \mathrm{d} \omega \\
& +\frac{r K}{2 \pi i} \int_{c-i \infty}^{c+i \infty}\left(S_{t}^{n}\right)^{-\omega} \int_{t}^{T} \frac{\left(\bar{S}_{t}^{n}(y)\right)^{\omega}}{\omega} \mathrm{e}^{\frac{1}{2} n^{2} \sigma^{2}\left(\omega^{2}+\alpha_{1}^{*} \omega-\alpha_{2}\right)(y-t)} \mathrm{d} y \mathrm{~d} \omega \\
& -\frac{q}{2 \pi i} \int_{c-i \infty}^{c+i \infty}\left(S_{t}^{n}\right)^{-\omega} \int_{t}^{T} \frac{\left(\bar{S}_{t}^{n}(y)\right)^{\omega+1}}{\omega+1} \mathrm{e}^{\frac{1}{2} n^{2} \sigma^{2}\left(\omega^{2}+\alpha_{1}^{*} \omega-\alpha_{2}\right)(y-t)} \mathrm{d} y \mathrm{~d} \omega
\end{aligned}
$$

Proof. Consider the non-homogeneous Black-Scholes partial differential equation for the American power put option with dividend yield given by

$$
\begin{aligned}
& \frac{\partial A_{p}^{n}\left(S_{t}^{n}, t\right)}{\partial t}+n\left(\frac{1}{2} \sigma^{2}(n-1)+(r-q)\right) S_{t}^{n} \frac{\partial A_{p}^{n}\left(S_{t}^{n}, t\right)}{\partial S_{t}^{n}} \\
& +\frac{1}{2}\left(\sigma n S_{t}^{n}\right)^{2} \frac{\partial^{2} A_{p}^{n}\left(S_{t}^{n}, t\right)}{\partial\left(S_{t}^{n}\right)^{2}}-r A_{p}^{n}\left(S_{t}^{n}, t\right)=f\left(S_{t}^{n}, t\right)
\end{aligned}
$$

where 


$$
f\left(S_{t}^{n}, t\right)= \begin{cases}-r K+q S_{t}^{n}, & \text { if } 0<S_{t}^{n} \leq \bar{S}_{t}^{n} \\ 0, & \text { if } S_{t}^{n}>\bar{S}_{t}^{n}\end{cases}
$$

on $(0, \infty) \times[0, T)$ and $\bar{S}_{t}^{n}$ the free boundary of the American power put option with dividend yield. The high contact condition is given by $A_{p}\left(S_{t}^{n}, T\right)=\phi\left(S_{t}^{n}\right)=\max \left(K-S_{t}^{n}, 0\right)=\left(K-S_{t}^{n}\right)^{+}$on $[0, \infty)$. The other conditions are given by (7) and (8). With the smooth pasting conditions given by

$$
A_{p}\left(\bar{S}_{t}^{n}, t\right)=K-\bar{S}_{t}^{n}
$$

and

$$
\frac{\partial A_{p}\left(\bar{S}_{t}^{n}, t\right)}{\partial \bar{S}_{t}^{n}}=-1
$$

The Mellin transform of (24) gives

$$
\frac{\partial \tilde{A}_{p}^{n}(\omega, t)}{\partial t}+\frac{n^{2} \sigma^{2}}{2}\left(\omega^{2}+\omega\left(1-\frac{n-1}{n}-\frac{2(r-q)}{n \sigma^{2}}\right)-\frac{2 r}{n^{2} \sigma^{2}}\right) \tilde{A}_{p}^{n}(\omega, t)=\tilde{f}(\omega, t)
$$

Putting $\alpha_{1}^{*}=\left(1-\frac{n-1}{n}-\frac{2(r-q)}{n \sigma^{2}}\right)$ and $\alpha_{2}=\frac{2 r}{n^{2} \sigma^{2}}$, (28) yields

$$
\frac{\partial \tilde{A}_{p}^{n}(\omega, t)}{\partial t}+\frac{n^{2} \sigma^{2}}{2}\left(\omega^{2}+\omega \alpha_{1}^{*}-\alpha_{2}\right) \tilde{A}_{p}^{n}(\omega, t)=\tilde{f}(\omega, t)
$$

where

$$
\tilde{f}(\omega, t)=\int_{0}^{\infty} f\left(S_{t}^{n}, t\right)\left(S_{t}^{n}\right)^{\omega-1} \mathrm{~d} S_{t}^{n}=\int_{0}^{\hat{S}_{t}^{n}}(-r K+q)\left(S_{t}^{n}\right)^{\omega-1} \mathrm{~d} S_{t}^{n}=\frac{-r K\left(\bar{S}_{t}^{n}\right)^{\omega}}{\omega}+\frac{q\left(\bar{S}_{t}^{n}\right)^{\omega+1}}{\omega+1}
$$

Following the same procedures for the case of non-dividend yield, the general solution to (30) is obtained as

$$
\begin{aligned}
\tilde{A}_{p}^{n}(\omega, t)= & \frac{K^{\omega+1}}{\omega(\omega+1)} \mathrm{e}^{\frac{1}{2} n^{2} \sigma^{2}\left(\omega^{2}+\alpha_{1}^{*} \omega-\alpha_{2}\right)(T-t)}+\int_{t}^{T} \frac{r K\left(\bar{S}_{y}^{n}\right)^{\omega}}{\omega} \mathrm{e}^{\frac{1}{2} n^{2} \sigma^{2}\left(\omega^{2}+\alpha_{1}^{*}\left(\omega-\alpha_{2}\right)(y-t)\right.} \mathrm{d} y \\
& -\int_{t}^{T} \frac{q\left(\bar{S}_{y}^{n}\right)^{\omega+1}}{\omega+1} \mathrm{e}^{\frac{1}{2} n^{2} \sigma^{2}\left(\omega^{2}+\alpha_{1}^{*} \omega-\alpha_{2}\right)(y-t)} \mathrm{d} y
\end{aligned}
$$

The Mellin inversion of (31) is given by

$$
\begin{aligned}
A_{p}^{n}\left(S_{t}^{n}, t\right)= & \frac{1}{2 \pi i} \int_{c-i \infty}^{c+i \infty} \frac{K^{\omega+1}}{\omega(\omega+1)} \mathrm{e}^{\frac{1}{2} n^{2} \sigma^{2}\left(\omega^{2}+\alpha_{1}^{*} \omega-\alpha_{2}\right)(T-t)}\left(S_{t}^{n}\right)^{-\omega} \mathrm{d} \omega \\
& +\frac{r K}{2 \pi i} \int_{c-i \infty}^{c+i \infty}\left(S_{t}^{n}\right)^{-\omega} \int_{t}^{T} \frac{\left(\bar{S}_{y}^{n}\right)^{\omega}}{\omega} \mathrm{e}^{\frac{1}{2} n^{2} \sigma^{2}\left(\omega^{2}+\alpha_{1}^{*} \omega-\alpha_{2}\right)(y-t)} \mathrm{d} y \mathrm{~d} \omega \\
& -\frac{q}{2 \pi i} \int_{c-i \infty}^{c+i \infty}\left(S_{t}^{n}\right)^{-\omega} \int_{t}^{T} \frac{\left(\bar{S}_{y}^{n}\right)^{\omega+1}}{\omega+1} \mathrm{e}^{\frac{1}{2} n^{2} \sigma^{2}\left(\omega^{2}+\alpha_{1}^{*} \omega-\alpha_{2}\right)(y-t)} \mathrm{d} y \mathrm{~d} \omega
\end{aligned}
$$

Equation (32) is the integral representation for the price of American power put option with dividend yield, where $\left(S_{t}^{n}, t\right) \in\{(0, \infty) \times[0, T]\}, c \in(0, \infty)$ and $\{\omega \in C \mid 0<\operatorname{Re}(\omega)<\infty\}$.

Remark 3

- The first term in (32) is the integral representation for the price of the European power put option (stems from the minimum guaranteed payoff of the American power put) with dividend yield and the last two terms denote the early exercise premium (the value attributable to the right of exercising the option early) for the American power put option with dividend yield denoted by $e_{p}^{n}\left(S_{t}^{n}, t\right)$. Therefore (32) becomes 


$$
A_{p}^{n}\left(S_{t}^{n}, t\right)=E_{p}^{n}\left(S_{t}^{n}, t\right)+e_{p}^{n}\left(S_{t}^{n}, t\right)
$$

where

$$
\begin{aligned}
E_{p}^{n}\left(S_{t}^{n}, t\right) & =\frac{1}{2 \pi i} \int_{c-i \infty}^{c+i \infty} \frac{K^{\omega+1}}{\omega(\omega+1)} \mathrm{e}^{\frac{1}{2} n^{2} \sigma^{2}\left(\omega^{2}+\alpha_{1}^{*} \omega-\alpha_{2}\right)(T-t)}\left(S_{t}^{n}\right)^{-\omega} \mathrm{d} \omega \\
e_{p}^{n}\left(S_{t}^{n}, t\right)= & \frac{r K}{2 \pi i} \int_{c-i \infty}^{c+i \infty}\left(S_{t}^{n}\right)^{-\omega} \int_{t}^{T} \frac{\left(\bar{S}_{y}^{n}\right)^{\omega}}{\omega} \mathrm{e}^{\frac{1}{2} n^{2} \sigma^{2}\left(\omega^{2}+\alpha_{1}^{*} \omega-\alpha_{2}\right)(y-t)} \mathrm{d} y \mathrm{~d} \omega \\
& -\frac{q}{2 \pi i} \int_{c-i \infty}^{c+i \infty}\left(S_{t}^{n}\right)^{-\omega} \int_{t}^{T} \frac{\left(\bar{S}_{y}^{n}\right)^{\omega+1}}{\omega+1} \mathrm{e}^{\frac{1}{2} n^{2} \sigma^{2}\left(\omega^{2}+\alpha_{1}^{*} \omega-\alpha_{2}\right)(y-t)} \mathrm{d} y \mathrm{~d} \omega
\end{aligned}
$$

- Setting $S_{t}^{n}=\bar{S}_{t}^{n}$ in (33) and using the smooth pasting conditions given by (26) and (27), we have the integral representation for the free boundary of the American power put option with dividend yield as

$$
\begin{aligned}
\bar{S}_{t}^{n}= & K-E_{p}^{n}\left(\bar{S}_{t}^{n}, t\right)-\frac{r K}{2 \pi i} \int_{c-i \infty}^{c+i \infty}\left(\bar{S}_{t}^{n}\right)^{-\omega} \int_{t}^{T} \frac{\left(\bar{S}_{y}^{n}\right)^{\omega}}{\omega} \mathrm{e}^{\frac{1}{2} n^{2} \sigma^{2}\left(\omega^{2}+\alpha_{1}^{*} \omega-\alpha_{2}\right)(y-t)} \mathrm{d} y \mathrm{~d} \omega \\
& +\frac{q}{2 \pi i} \int_{c-i \infty}^{c+i \infty}\left(\bar{S}_{t}^{n}\right)^{-\omega} \int_{t}^{T} \frac{\left(\bar{S}_{y}^{n}\right)^{\omega+1}}{\omega+1} \mathrm{e}^{\frac{1}{2} n^{2} \sigma^{2}\left(\omega^{2}+\alpha_{1}^{*} \omega-\alpha_{2}\right)(y-t)} \mathrm{d} y \mathrm{~d} \omega
\end{aligned}
$$

where

$$
E_{p}^{n}\left(\hat{S}_{t}^{n}, t\right)=\frac{1}{2 \pi i} \int_{c-i \infty}^{c+i \infty} \frac{K^{\omega+1}}{\omega(\omega+1)} \mathrm{e}^{\frac{1}{2} n^{2} \sigma^{2}\left(\omega^{2}+\alpha_{1}^{*} \omega-\alpha_{2}\right)(T-t)}\left(\bar{S}_{t}^{n}\right)^{-\omega} \mathrm{d} \omega
$$

From Remark 3, we have the following results.

Theorem 4 The American power put option $A_{p}^{n}\left(S_{t}^{n}, t\right)$ which pays dividend yield satisfies the decomposition

$$
\begin{aligned}
A_{p}^{n}\left(S_{t}^{n}, t\right)= & E_{p}^{n}\left(S_{t}^{n}, t\right)+\frac{r K}{2 \pi i} \int_{c-i \infty}^{c+i \infty}\left(S_{t}^{n}\right)^{-\omega} \int_{t}^{T} \frac{\left(\bar{S}_{y}^{n}\right)^{\omega}}{\omega} \mathrm{e}^{\frac{1}{2} n^{2} \sigma^{2}\left(\omega^{2}+\alpha_{1}^{*} \omega-\alpha_{2}\right)(y-t)} \mathrm{d} y \mathrm{~d} \omega \\
& -\frac{q}{2 \pi i} \int_{c-i \infty}^{c+i \infty}\left(S_{t}^{n}\right)^{-\omega} \int_{t}^{T} \frac{\left(\bar{S}_{y}^{n}\right)^{\omega+1}}{\omega+1} \mathrm{e}^{\frac{1}{2} n^{2} \sigma^{2}\left(\omega^{2}+\alpha_{1}^{*} \omega-\alpha_{2}\right)(y-t)} \mathrm{d} y \mathrm{~d} \omega
\end{aligned}
$$

where $\alpha_{1}^{*}=\left(1-\frac{n-1}{n}-\frac{2(r-q)}{n \sigma^{2}}\right)$ and $\alpha_{2}=\frac{2 r}{n^{2} \sigma^{2}}, \quad\left(S_{t}^{n}, t\right) \in\{(0, \infty) \times[0, T)\}, c \in(0, \infty)$ and $\{\omega \in C \mid 0<\operatorname{Re}(\omega)<\infty\}$.

Theorem 5 Using the smooth pasting conditions given by $A_{p}\left(\bar{S}_{t}^{n}, t\right)=K-\bar{S}_{t}^{n}$ and $\frac{\partial A_{p}\left(\bar{S}_{t}^{n}, t\right)}{\partial \bar{S}_{t}^{n}}=-1$. Then the free boundary formulation of the American power put option with dividend yield is given by

$$
\begin{gathered}
\bar{S}_{t}^{n}=K-E_{p}^{n}\left(\bar{S}_{t}^{n}, t\right) \\
-\frac{r K}{2 \pi i} \int_{c-i \infty}^{c+i \infty}\left(\bar{S}_{t}^{n}\right)^{-\omega} \int_{t}^{T} \frac{\left(\bar{S}_{y}^{n}\right)^{\omega}}{\omega} \mathrm{e}^{\frac{1}{2} n^{2} \sigma^{2}\left(\omega^{2}+\alpha_{1}^{*} \omega-\alpha_{2}\right)(y-t)} \mathrm{d} y \mathrm{~d} \omega \\
+\frac{q}{2 \pi i} \int_{c-i \infty}^{c+i \infty}\left(\bar{S}_{t}^{n}\right)^{-\omega} \int_{t}^{T} \frac{\left(\bar{S}_{y}^{n}\right)^{\omega+1}}{\omega+1} \mathrm{e}^{\frac{1}{2} n^{2} \sigma^{2}\left(\omega^{2}+\alpha_{1}^{*} \omega-\alpha_{2}\right)(y-t)} \mathrm{d} y \mathrm{~d} \omega
\end{gathered}
$$

The following results present some special cases of (20) and (32).

Theorem 6 If $\tau \rightarrow T-t$ and $n=1$, then

(i) The integral representation for the American power put option which pays no dividend yield (20) reduces to the integral equation derived by Kim [6] for the price of the plain American put option given by 


$$
A_{p}\left(S_{\tau}, \tau\right)=E_{p}\left(S_{\tau}, \tau\right)+\int_{0}^{\tau} r K \mathrm{e}^{-r \eta} N\left(-d_{\eta}\right) \mathrm{d} \eta
$$

where

$$
d_{\eta}=\frac{\ln \left(\frac{S_{\tau}}{\hat{S}_{(\tau-\eta)}}\right)+\left(r-\frac{\sigma^{2}}{2}\right) \eta}{\sigma \sqrt{\eta}}
$$

(ii) The free boundary for the American power put option which pays no dividend yield (22) reduces to the integral equation derived by Kim [6] for the price of the plain American put option given by

$$
\hat{S}_{\tau}=K-E_{p}\left(\hat{S}_{\tau}, \tau\right)-\int_{0}^{\tau} r K \mathrm{e}^{-r \eta} N\left(-d_{\eta}\right) \mathrm{d} \eta
$$

where

$$
d_{\eta}=\frac{\ln \left(\frac{\hat{S}_{\tau}}{\hat{S}_{(\tau-\eta)}}\right)+\left(r-\frac{\sigma^{2}}{2}\right) \eta}{\sigma \sqrt{\eta}}
$$

Proof. Setting $n=1$ and $\tau=T-t$ in (20) yields

$$
\begin{aligned}
A_{p}\left(S_{\tau}, \tau\right)= & \frac{1}{2 \pi i} \int_{c-i \infty}^{c+i \infty} \frac{K^{\omega+1}}{\omega(\omega+1)} \mathrm{e}^{\frac{1}{2} \sigma^{2}\left(\omega^{2}+\alpha_{1} \omega-\alpha_{2}\right)(\tau)}\left(S_{\tau}\right)^{-\omega} \mathrm{d} \omega \\
& +\frac{r K}{2 \pi i} \int_{c-i \infty}^{c+i \infty}\left(S_{\tau}\right)^{-\omega} \int_{0}^{\tau} \frac{\left(\hat{S}_{y}\right)^{\omega}}{\omega} \mathrm{e}^{\frac{1}{2} \sigma^{2}\left(\omega^{2}+\alpha_{1} \omega-\alpha_{2}\right)(\tau-y)} \mathrm{d} y \mathrm{~d} \omega
\end{aligned}
$$

where $\alpha_{1}=\left(1-\frac{2 r}{\sigma^{2}}\right)$ and $\alpha_{2}=\frac{2 r}{\sigma^{2}}$. Equation (39) can be be written as

$$
A_{p}\left(S_{\tau}, \tau\right)=E_{p}\left(S_{\tau}, \tau\right)+e_{p}\left(S_{\tau}, \tau\right)
$$

where $E_{p}\left(S_{\tau}, \tau\right)$ and $e_{p}\left(S_{\tau}, \tau\right)$ denote the price of the European put option with no dividend yield and free boundary for the American put option with no dividend yield respectively. Let

$$
e_{p}\left(S_{\tau}, \tau\right)=\int_{0}^{\tau} \Omega\left(S_{\tau}, \hat{S}_{y}, \tau, y\right) \mathrm{d} y
$$

where

$$
\Omega\left(S_{\tau}, \hat{S}_{y}, \tau, y\right)=\frac{1}{2 \pi i} \int_{c-i \infty}^{c+i \infty} \hat{f}(\omega, y) \hat{\xi}(\omega, y) S_{\tau}^{-\omega} \mathrm{d} \omega
$$

The early exercise function is given by

$$
f\left(S_{\tau}, \tau\right)= \begin{cases}r K, & \text { if } S_{\tau} \in\left(0, \hat{S}_{y}\right] \\ 0, & \text { if } S_{\tau}>S_{y}\end{cases}
$$

and

$$
\hat{\xi}(\omega, y)=\mathrm{e}^{\frac{1}{2} \sigma^{2}\left(\omega^{2}+\alpha_{1} \omega-\alpha_{2}\right)(\tau-y)}
$$

Using the convolution property of the Mellin transform, (42) becomes

$$
\Omega\left(S_{\tau}, \hat{S}_{y}, \tau, y\right)=\int_{0}^{y} f(v, y) \xi\left(\frac{S_{\tau}}{v}, y\right) \frac{1}{v} \mathrm{~d} v
$$

Substituting the value of the early exercise function $f(v, y)$ from (43) and 


$$
\xi\left(S_{\tau}\right)=\mathrm{e}^{-\frac{\sigma^{2}}{2}(\tau-y)\left(\frac{\alpha_{2}+1}{2}\right)^{2}} \frac{S_{\tau}^{\frac{1-\alpha_{2}}{2}}}{\sigma \sqrt{2 \pi(\tau-y)}} \mathrm{e}^{-\frac{1}{2}\left(\frac{\ln S_{\tau}}{\sigma \sqrt{\tau-y}}\right)^{2}}
$$

into (45), we have that

$$
\Omega\left(S_{\tau}, \hat{S}_{y}, \tau, y\right)=r K \int_{0}^{\hat{S}_{y}} \frac{\mathrm{e}^{-\frac{\sigma^{2}}{2}(\tau-y)\left(\frac{\alpha_{2}+1}{2}\right)^{2}}}{v^{\left(1+\frac{1-\alpha_{2}}{2}\right)}} \frac{S_{\tau}^{\frac{1-\alpha_{2}}{2}}}{\sigma \sqrt{2 \pi(\tau-y)}} \mathrm{e}^{-\frac{1}{2}\left(\frac{\ln S_{\tau}}{\sigma \sqrt{\tau-y}}\right)^{2}} \mathrm{~d} v
$$

Using the transformation given by

$$
\lambda=\frac{1}{\sigma \sqrt{\tau-y}}\left(\ln \left(\frac{S_{\tau}}{v}\right)-\sigma^{2}(\tau-y) \frac{1-\alpha_{2}}{2}\right)
$$

(47) becomes

$$
\Omega\left(S_{\tau}, \hat{S}_{y}, \tau, y\right)=r K \mathrm{e}^{-r(\tau-y)} \frac{1}{\sqrt{2 \pi}} \int_{d_{y}}^{\infty} \mathrm{e}^{-\frac{\lambda^{2}}{2}} \mathrm{~d} \lambda=r K \mathrm{e}^{-r(\tau-y)} N\left(-d_{y}\right)
$$

Substituting (49) into (41) we have the early exercise premium for the American put option with non-dividend yield as

$$
e_{p}\left(S_{\tau}, \tau\right)=r K \int_{0}^{\tau} \mathrm{e}^{-r(\tau-y)} N\left(-d_{y}\right) \mathrm{d} y
$$

where

$$
d_{y}=\frac{\ln \left(\frac{S_{\tau}}{\hat{S}_{y}}\right)+\left(r-\frac{\sigma^{2}}{2}\right)(\tau-y)}{\sigma \sqrt{\tau-y}}
$$

Setting $\eta=\tau-y$, then (50) becomes

$$
e_{p}\left(S_{\tau}, \tau\right)=\int_{0}^{\tau} r K \mathrm{e}^{-r \eta} N\left(-d_{\eta}\right) \mathrm{d} \eta
$$

where

$$
d_{\eta}=\frac{\ln \left(\frac{S_{\tau}}{\hat{S}_{(\tau-\eta)}}\right)+\left(r-\frac{\sigma^{2}}{2}\right) \eta}{\sigma \sqrt{\eta}}
$$

Substituting (52) into (40) we get the integral equation (35) obtained by Kim [6] as

$$
A_{p}\left(S_{\tau}, \tau\right)=E_{p}\left(S_{\tau}, \tau\right)+\int_{0}^{\tau} r K \mathrm{e}^{-r \eta} N\left(-d_{\eta}\right) \mathrm{d} \eta
$$

Hence (i) is established. For the second reduction, setting $S_{\tau}=\hat{S}_{\tau}$ in the last integral equation above and using the smooth pasting conditions given by $A_{p}\left(\hat{S}_{\tau}, \tau\right)=K-\hat{S}_{\tau}$ and $\frac{\partial A_{p}\left(\hat{S}_{\tau}, \tau\right)}{\partial \hat{S}_{\tau}}$, we obtain the free boundary $\hat{S}_{\tau}$ of the American put option which pay no dividend yield (37) derived by Kim [6] as

$$
\hat{S}_{\tau}=K-E_{p}\left(\hat{S}_{\tau}, \tau\right)-\int_{0}^{\tau} r K \mathrm{e}^{-r \eta} N\left(-\hat{d}_{\eta}\right) \mathrm{d} \eta
$$

where 


$$
\hat{d}_{\eta}=\frac{\ln \left(\frac{\hat{S}_{\tau}}{\hat{S}_{(\tau-\eta)}}\right)+\left(r-\frac{\sigma^{2}}{2}\right) \eta}{\sigma \sqrt{\eta}}
$$

Theorem 7 If $t \rightarrow T$, then the optimal exercise boundary $\bar{S}_{t}$ of the American power put option with $n=1$ with dividend yield is given by

$$
\lim _{t \rightarrow T} \bar{S}_{t}=\min \left(K, \frac{r K}{q}\right)
$$

Proof. Let $\tau=T-t$ and $n=1$, (34) becomes

$$
\begin{aligned}
\bar{S}_{\tau}= & K-E_{p}\left(\bar{S}_{\tau}, \tau\right)-\frac{r K}{2 \pi i} \int_{c-i \infty}^{c+i \infty}\left(\bar{S}_{\tau}\right)^{-\omega} \int_{0}^{\tau} \frac{\left(\bar{S}_{y}\right)^{\omega}}{\omega} \mathrm{e}^{\frac{1}{2} \sigma^{2}\left(\omega^{2}+\alpha_{1}^{*} \omega-\alpha_{2}\right)(\tau-y)} \mathrm{d} y \mathrm{~d} \omega \\
& +\frac{q}{2 \pi i} \int_{c-i \infty}^{c+i \infty}\left(\bar{S}_{\tau}\right)^{-\omega} \int_{0}^{\tau} \frac{\left(\bar{S}_{y}\right)^{\omega+1}}{\omega+1} \mathrm{e}^{\frac{1}{2} \sigma^{2}\left(\omega^{2}+\alpha_{1}^{*} \omega-\alpha_{2}\right)(\tau-y)} \mathrm{d} y \mathrm{~d} \omega
\end{aligned}
$$

where $\alpha_{1}^{*}=\left(1-\frac{2(r-q)}{\sigma^{2}}\right)$ and $\alpha_{2}=\frac{2 r}{\sigma^{2}}$. Factorizing and rearranging, (54) becomes

$$
\bar{S}_{\tau}=K\left(\frac{1+\mathrm{e}^{-r \tau}\left(N\left(d_{2}\left(\bar{S}_{\tau}, K, \tau\right)\right)-1\right)-r I_{\tau}}{1+\mathrm{e}^{-q \tau}\left(N\left(d_{1}\left(\bar{S}_{\tau}, K, \tau\right)\right)-1\right)-q J_{\tau}}\right)
$$

where

$$
\begin{gathered}
d_{1}\left(\bar{S}_{\tau}, K, \tau\right)=\frac{\ln \left(\frac{\bar{S}_{\tau}}{K}\right)+\left(r-q+\frac{1}{2} \sigma^{2}\right) \tau}{\sigma \sqrt{\tau}} \\
d_{2}\left(\bar{S}_{\tau}, K, \tau\right)=\frac{\ln \left(\frac{\bar{S}_{\tau}}{K}\right)+\left(r-q-\frac{1}{2} \sigma^{2}\right) \tau}{\sigma \sqrt{\tau}} \\
I_{\tau}=\frac{1}{2 \pi i} \int_{c-i \infty}^{c+i \infty}\left(\bar{S}_{\tau}\right)^{-\omega} \int_{0}^{\tau} \frac{\left(\bar{S}_{y}\right)^{\omega}}{\omega} \mathrm{e}^{\frac{1}{2} \sigma^{2}\left(\omega^{2}+\alpha_{1}^{*} \omega-\alpha_{2}\right)(\tau-y)} \mathrm{d} y \mathrm{~d} \omega
\end{gathered}
$$

and

$$
J_{\tau}=\frac{1}{2 \pi i} \int_{c-i \infty}^{c+i \infty}\left(\bar{S}_{\tau}\right)^{-\omega} \int_{0}^{\tau} \frac{\left(\bar{S}_{y}\right)^{\omega+1}}{\omega+1} \mathrm{e}^{\frac{1}{2} \sigma^{2}\left(\omega^{2}+\alpha_{1}^{*} \omega-\alpha_{2}\right)(\tau-y)} \mathrm{d} y \mathrm{~d} \omega
$$

Notice first that critical stock price is bounded from above i.e. $\bar{S}_{\tau} \leq K, \forall \tau>0$. Taking the limits of (56) and (57) as $\tau \rightarrow 0^{+}$, we have that

$$
\lim _{\tau \rightarrow 0^{+}} d_{1}\left(\bar{S}_{\tau}, K, \tau\right)= \begin{cases}0, & \text { for } \bar{S}_{\tau}\left(0^{+}\right)=K \\ -\infty, & \text { for } \bar{S}_{\tau}\left(0^{+}\right)<K\end{cases}
$$

and

$$
\lim _{\tau \rightarrow 0^{+}} d_{2}\left(\bar{S}_{\tau}, K, \tau\right)= \begin{cases}0, & \text { for } \bar{S}_{\tau}\left(0^{+}\right)=K \\ -\infty, & \text { for } \bar{S}_{\tau}\left(0^{+}\right)<K\end{cases}
$$


respectively. If

$$
\lim _{\tau \rightarrow 0^{+}} \bar{S}_{\tau}=K
$$

We have

$$
\lim _{\tau \rightarrow 0^{+}} N\left(d_{1}\left(\bar{S}_{\tau}, K, \tau\right)\right)=\lim _{\tau \rightarrow 0^{+}} N\left(d_{2}\left(\bar{S}_{\tau}, K, \tau\right)\right)=\frac{1}{2}
$$

Using (63), the limit of (55) is obtained as

$$
\begin{gathered}
\lim _{\tau \rightarrow 0^{+}} \bar{S}_{\tau}=K \lim _{\tau \rightarrow 0^{+}}\left(\frac{1+\mathrm{e}^{-r \tau}\left(N\left(d_{2}\left(\bar{S}_{\tau}, K, \tau\right)\right)-1\right)-r I_{\tau}}{1+\mathrm{e}^{-q \tau}\left(N\left(d_{1}\left(\bar{S}_{\tau}, K, \tau\right)\right)-1\right)-q J_{\tau}}\right) \\
\lim _{\tau \rightarrow 0^{+}} \bar{S}_{\tau}=K\left(\frac{\frac{1}{2}-\lim _{\tau \rightarrow 0^{+}} r I_{\tau}}{\frac{1}{2}-\lim _{\tau \rightarrow 0^{+}} q J_{\tau}}\right)
\end{gathered}
$$

Since

$$
\lim _{\tau \rightarrow 0^{+}} I_{\tau}=0
$$

and

$$
\lim _{\tau \rightarrow 0^{+}} J_{\tau}=0
$$

Then (64) becomes

$$
\lim _{\tau \rightarrow 0^{+}} \frac{\bar{S}_{\tau}}{K}=1
$$

If

$$
\lim _{\tau \rightarrow 0^{+}} \bar{S}_{\tau}<K
$$

We have that

$$
\lim _{\tau \rightarrow 0^{+}} \frac{\bar{S}_{\tau}}{K}=\left(\frac{r}{q}\right) \lim _{\tau \rightarrow 0^{+}}\left(\frac{I_{\tau}}{J_{\tau}}\right)
$$

The first integral $I_{\tau}$ can also be written as

$$
I_{\tau}=\int_{0}^{\tau} \frac{1}{2 \pi i} \int_{c-i \infty}^{c+i \infty}\left(\bar{S}_{\tau}\right)^{-\omega} \frac{\left(\bar{S}_{y}\right)^{\omega}}{\omega} \mathrm{e}^{\frac{1}{2} \sigma^{2}\left(\omega^{2}+\alpha_{1}^{*} \omega-\alpha_{2}\right)(\tau-y)} \mathrm{d} y \mathrm{~d} \omega
$$

Applying the residue theorem of complex number given by

$$
\int_{\mathcal{C}} f(w) \mathrm{d} w=2 \pi i \sum_{j=0}^{k} \operatorname{Res}_{w=w_{j}} f(w), w \in \mathcal{C}
$$

Then the inner integral in (67) becomes

$$
\frac{1}{2 \pi i} \int_{c-i \infty}^{c+i \infty}\left(\bar{S}_{\tau}\right)^{-\omega} \frac{\left(\bar{S}_{y}\right)^{\omega}}{\omega} \mathrm{e}^{\frac{1}{2} \sigma^{2}\left(\omega^{2}+\alpha_{1}^{*} \omega-\alpha_{2}\right)(\tau-y)} \mathrm{d} y \mathrm{~d} \omega=\mathrm{e}^{-r(\tau-y)}
$$

Substituting (69) into (67) yields

$$
I_{\tau}=\frac{-\left(1-\mathrm{e}^{-r \tau}\right)}{r}
$$


Similarly,

$$
J_{\tau}=\frac{-\left(1-\mathrm{e}^{-q \tau}\right)}{q}
$$

Substituting (70) and (71) into (66) for $q \leq r$, we have that

$$
\lim _{\tau \rightarrow 0^{+}} \frac{\bar{S}_{\tau}}{K}=\left(\frac{r}{q}\right) \lim _{\tau \rightarrow 0^{+}}\left(\frac{\frac{1-\mathrm{e}^{-r \tau}}{r}}{\frac{r-\mathrm{e}^{-q \tau}}{q}}\right)=\lim _{\tau \rightarrow 0^{+}}\left(\frac{1-\mathrm{e}^{-r \tau}}{1-\mathrm{e}^{-q \tau}}\right)=1
$$

Using the l'Hospital rule, for $q>r$, (64) becomes

$$
\lim _{\tau \rightarrow 0^{+}} \frac{\bar{S}_{\tau}}{K}=\frac{r}{q}
$$

Combining (72) and (73)

$$
\lim _{\tau \rightarrow 0^{+}} \bar{S}_{\tau}=\min \left(K, \frac{r K}{q}\right)
$$

Hence (53) is established.

\section{Remark 4}

The above results confirm the formula of Kim and $\mathrm{Yu}$ [6]

Theorem 8 If the underlying asset price follows a lognormal diffusion process and the interest rate is a positive constant, then the optimal exercise boundary of the American power put option with $n=1$ at maturity is given by

$$
\lim _{\tau \rightarrow} \bar{S}_{\tau}= \begin{cases}\frac{r K}{q}, & \text { for } q>r \\ K, & \text { for } q \leq r\end{cases}
$$

Proof. Let $t \mapsto \tau=t-T$. In order to investigate the behaviour of the optimal exercise boundary $\bar{S}_{\tau}$ of the American power put option with $n=1$ near maturity, we consider (55) which is of the form

$$
\bar{S}_{\tau}=K\left(\frac{1+\mathrm{e}^{-r \tau}\left(N\left(d_{2}\left(\bar{S}_{\tau}, K, \tau\right)\right)-1\right)-r I_{\tau}}{1+\mathrm{e}^{-q \tau}\left(N\left(d_{1}\left(\bar{S}_{\tau}, K, \tau\right)\right)-1\right)-q J_{\tau}}\right)
$$

If $q>r$, the limit of the right hand side of (55) as $\tau \rightarrow 0$ can be evaluated using the l'Hospital's rule we have that

$$
\lim _{\tau \rightarrow 0} \bar{S}_{\tau}=\frac{r K}{q}
$$

If $q \leq r$, the limit of the right hand side of (55) as $\tau \rightarrow 0$ is obtained directly as

$$
\lim _{\tau \rightarrow 0} \bar{S}_{\tau}=K
$$

Combining (75) and (76), we have the optimal exercise boundary of the American power put option with $n=1$ at maturity given by

$$
\lim _{\tau \rightarrow} \bar{S}_{\tau}= \begin{cases}\frac{r K}{q}, & \text { for } q>r \\ K, & \text { for } q \leq r\end{cases}
$$

Hence (74) is established.

\section{Remark 5}


From (75), we notice that when $q>r$ the American put can have a positive value at expiration given that it has not been exercised earlier. This indicates that large dividend payouts reduce the incentives of early exercise.

From (76), we deduce that when $q \leq r$ the American put will have a zero payoff at expiration even if it has not been exercised earlier. This is because it is not possible for the underlying asset price at expiration to fall below $K$ without crossing the exercise boundary at an earlier time.

Theorem 9 The integral representation for the price of the American power put option which pays dividend yield given by (32) can be reduced to integral representation derived by Kim [6].

$$
\begin{aligned}
A_{p}\left(S_{\tau}, \tau\right)= & E_{p}\left(S_{\tau}, \tau\right)+\int_{0}^{\tau} r K \mathrm{e}^{-r(\tau-\eta)} N\left(-d_{2}\left(S_{\tau}, \bar{S}_{\eta}, \tau-\eta\right)\right) \mathrm{d} \eta \\
& -\int_{0}^{\tau} q S_{\tau} \mathrm{e}^{-r(\tau-\eta)} N\left(-d_{1}\left(S_{\tau}, \bar{S}_{\eta}, \tau-\eta\right)\right) \mathrm{d} \eta
\end{aligned}
$$

where

$$
\begin{gathered}
d_{1}\left(S_{\tau}, \bar{S}_{\eta}, \tau-\eta\right)=\frac{\ln \left(\frac{S_{\tau}}{\bar{S}_{(\tau-\eta)}}\right)+\left(r-q+\frac{\sigma^{2}}{2}\right)(\tau-\eta)}{\sigma \sqrt{\tau-\eta}} \\
d_{2}\left(S_{\tau}, \bar{S}_{\eta}, \tau-\eta\right)=\frac{\ln \left(\frac{S_{\tau}}{\bar{S}_{(\tau-\eta)}}\right)+\left(r-q-\frac{\sigma^{2}}{2}\right)(\tau-\eta)}{\sigma \sqrt{\tau-\eta}} \\
\tau=T-t \\
S_{\tau} \leq \bar{S}_{\tau}
\end{gathered}
$$

Proof. Setting $t \mapsto \tau=T-t$, then (32) becomes

$$
\begin{aligned}
A_{p}^{n}\left(S_{\tau}^{n}, \tau\right)= & E_{p}^{n}\left(S_{\tau}^{n}, \tau\right)+\frac{r K}{2 \pi i} \int_{c-i \infty}^{c+i \infty}\left(S_{\tau}^{n}\right)^{-\omega} \int_{0}^{\tau} \frac{\left(\bar{S}_{y}^{n}\right)^{\omega}}{\omega} \mathrm{e}^{\frac{1}{2} n^{2} \sigma^{2}\left(\omega^{2}+\alpha_{1}^{*} \omega-\alpha_{2}\right)(\tau-y)} \mathrm{d} y \mathrm{~d} \omega \\
& -\frac{q}{2 \pi i} \int_{c-i \infty}^{c+i \infty}\left(S_{t}^{n}\right)^{-\omega} \int_{0}^{\tau} \frac{\left(\bar{S}_{y}^{n}\right)^{\omega+1}}{\omega+1} \mathrm{e}^{\frac{1}{2} n^{2} \sigma^{2}\left(\omega^{2}+\alpha_{1}^{*} \omega-\alpha_{2}\right)(\tau-y)} \mathrm{d} y \mathrm{~d} \omega
\end{aligned}
$$

where $\alpha_{1}^{*}=\left(1-\frac{n-1}{n}-\frac{2(r-q)}{n \sigma^{2}}\right)$ and $\alpha_{2}=\frac{2 r}{n^{2} \sigma^{2}}, \quad\left(S_{\tau}^{n}, \tau\right) \in\{(0, \infty) \times[0, T]\}, c \in(0, \infty)$ and $\{\omega \in C \mid 0<\operatorname{Re}(\omega)<\infty\}$.

Using the procedures of [3], (78) can be written as

$$
A_{p}^{n}\left(S_{\tau}^{n}, \tau\right)=E_{p}^{n}\left(S_{\tau}^{n}, \tau\right)-\int_{0}^{\tau} \frac{1}{2 \pi i} \int_{c-i \infty}^{c+i \infty} \tilde{f}(\omega, y) \tilde{\xi}(\omega, y)\left(S_{\tau}^{n}\right)^{-\omega} \mathrm{d} \omega \mathrm{d} y
$$

with the Mellin transforms of $f\left(S_{\tau}^{n}, y\right)$ and $\xi\left(S_{\tau}^{n}, y\right)$ given by

$$
\begin{gathered}
\tilde{f}(\omega, y)=\frac{-r K\left(\bar{S}_{y}^{n}\right)^{\omega}}{\omega}+\frac{q}{\omega+1}\left(\bar{S}_{y}^{n}\right)^{\omega+1} \\
\tilde{\xi}(\omega, y)=\mathrm{e}^{\frac{1}{2} n^{2} \sigma^{2}\left(\omega^{2}+\alpha_{1}^{*} \omega-\alpha_{2}\right)(\tau-y)}
\end{gathered}
$$

respectively. Using the convolution theorem of the Mellin transform we have that

$$
A_{p}^{n}\left(S_{\tau}^{n}, \tau\right)=E_{p}^{n}\left(S_{\tau}^{n}, \tau\right)-\int_{0}^{\tau} \int_{0}^{\infty} f(v, y) \xi\left(\frac{S_{\tau}^{n}}{v}, y\right) \frac{1}{v} \mathrm{~d} v \mathrm{~d} y
$$

The price of the American power put option which pays dividend yield can be expressed as 


$$
A_{p}^{n}\left(S_{\tau}^{n}, \tau\right)=E_{p}^{n}\left(S_{\tau}^{n}, \tau\right)-\int_{0}^{\tau} I\left(S_{\tau}^{n}, y\right) \mathrm{d} y
$$

The integral $I\left(S_{\tau}^{n}, y\right)$ is evaluated as follows

$$
\begin{gathered}
I\left(S_{\tau}, y\right)=\int_{0}^{\infty} f(v, y) \xi\left(\frac{S_{\tau}^{n}}{v}, y\right) \frac{1}{v} \mathrm{~d} v \\
I\left(S_{\tau}^{n}, y\right)=-r K \mathrm{e}^{-\rho_{1}\left(\left(\rho_{2}^{*}\right)^{2}+\alpha_{2}\right)} \frac{\left(S_{\tau}^{n}\right)^{\rho_{2}^{*}}}{\sigma \sqrt{2 \pi(\tau-y)}} \int_{0}^{\bar{S}_{y}^{n}} \frac{1}{v^{\rho_{2}+1}} \mathrm{e}^{-\frac{1}{2}\left(\frac{\ln \frac{S_{\tau}^{n}}{v}}{\sigma \sqrt{\tau-y}}\right)^{2}} \mathrm{~d} v \\
+q \mathrm{e}^{-\rho_{1}\left(\left(\rho_{2}^{*}\right)^{2}+\alpha_{2}\right)} \frac{\left(S_{\tau}^{n}\right)^{\rho_{2}^{*}}}{\sigma \sqrt{2 \pi(\tau-y)}} \int_{0}^{\bar{S}_{y}^{n}} \frac{1}{v^{\rho_{2}^{*}}} \mathrm{e}^{-\frac{1}{2}\left(\frac{\ln \frac{S_{\tau}^{n}}{\sigma}}{\sigma \sqrt{\tau-y}}\right)^{2}} \mathrm{~d} v
\end{gathered}
$$

where $\rho_{1}=\frac{n^{2} \sigma^{2}}{2}(\tau-y), \rho_{2}^{*}=\frac{\alpha_{1}^{*}}{2}=\frac{1}{2}\left(1-\frac{n-1}{n}-\frac{2(r-q)}{n \sigma^{2}}\right)$ and $\alpha_{2}=\frac{2 r}{n^{2} \sigma^{2}}$. Using the following variables transformation given by

$$
\lambda_{1}=\frac{1}{\sqrt{\tau-y}}\left(\ln \left(\frac{S_{\tau}^{n}}{v}\right)-\rho_{2} n^{2} \sigma^{2}(\tau-y)\right)
$$

and

$$
\lambda_{2}=\frac{1}{\sqrt{\tau-y}}\left(\ln \left(\frac{S_{\tau}^{n}}{v}\right)-\left(\rho_{2}-1\right) n^{2} \sigma^{2}(\tau-y)\right)
$$

For the first and second integrals in (83) respectively, we have that

$$
\begin{aligned}
I\left(S_{\tau}^{n}, y\right)= & -r K \mathrm{e}^{-r(\tau-y)} N\left(-d_{2, n}\left(S_{\tau}^{n}, \bar{S}_{y}^{n}, \tau-y\right)\right) \\
& +q \mathrm{e}^{\left(r(n-1)-n q+\frac{1}{2} n(n-1) \sigma^{2}\right)(\tau-y)} N\left(-d_{1, n}\left(S_{\tau}^{n}, \bar{S}_{y}^{n}, \tau-y\right)\right)
\end{aligned}
$$

Substituting (84) into (82) yields

$$
\begin{aligned}
A_{p}^{n}\left(S_{\tau}^{n}, \tau\right)= & E_{p}^{n}\left(S_{\tau}^{n}, \tau\right)+\int_{0}^{\tau} r K \mathrm{e}^{-r(\tau-y)} N\left(-d_{2, n}\left(S_{\tau}^{n}, \bar{S}_{y}^{n}, \tau-y\right)\right) \mathrm{d} y \\
& -\int_{0}^{\tau} q \mathrm{e}^{\left(r(n-1)-n q+\frac{1}{2} n(n-1) \sigma^{2}\right)(\tau-y)} N\left(-d_{1, n}\left(S_{\tau}^{n}, \bar{S}_{y}^{n}, \tau-y\right)\right) \mathrm{d} y
\end{aligned}
$$

By changing $y=\eta$, (85) becomes

$$
\begin{aligned}
A_{p}^{n}\left(S_{\tau}^{n}, \tau\right)= & E_{p}^{n}\left(S_{\tau}^{n}, \tau\right)+\int_{0}^{\tau} r K \mathrm{e}^{-r(\tau-\eta)} N\left(-d_{2, n}\left(S_{\tau}^{n}, \bar{S}_{\eta}^{n}, \tau-\eta\right)\right) \mathrm{d} \eta \\
& -\int_{0}^{\tau} q \mathrm{e}^{\left(r(n-1)-n q+\frac{1}{2} n(n-1) \sigma^{2}\right)(\tau-\eta)} N\left(-d_{1, n}\left(S_{\tau}^{n}, \bar{S}_{\eta}^{n}, \tau-\eta\right)\right) \mathrm{d} \eta
\end{aligned}
$$

Hence by setting $n=1$, this proves (77).

\section{Application of the Results to Perpetual American Power Put Option Valuation}

Now, we apply the results generated for the integral equations in (20) and (32) to power options which have no expiry date. The following results shows the derivation of the expression for the free boundary of perpetual the American power put option and its closed form solution for both non-dividend and dividend yields, using the Mellin transform method. 
Theorem 10 (Non-Dividend Yield) If $T \rightarrow \infty$ and $0<\operatorname{Re}(\omega)<\alpha_{2}$, then the free boundary of the perpetual American power put option is given by

$$
\hat{S}_{\infty}^{n}=\hat{S}_{\infty}^{n}(t)=K \frac{\alpha_{2}}{\left(\alpha_{2}+1\right)}
$$

and the price of the perpetual American power option becomes

$$
A_{\infty}^{n}\left(S_{t}^{n}, t\right)=\left(K-\hat{S}_{\infty}^{n}\right)\left(\frac{S_{t}^{n}}{\hat{S}_{\infty}^{n}}\right)^{-\alpha_{2}} \text { for } \hat{S}_{\infty}^{n}<S_{t}^{n}
$$

where

$$
\alpha_{2}=\frac{2 r}{n^{2} \sigma^{2}}
$$

Proof. The integral representation for the price of the American power put option which pays no dividend yield given by (20) can be expressed as

$$
A_{p}^{n}\left(S_{t}, t\right)=E_{p}^{n}\left(S_{t}^{n}, t\right)+P_{1}^{n}\left(S_{t}^{n}, t\right)
$$

where $E_{p}^{n}\left(S_{t}, t\right)$ and $P_{1}^{n}\left(S_{t}^{n}, t\right)$ are given by

$$
E_{p}^{n}\left(S_{t}, t\right)=K \mathrm{e}^{-r(T-t)} N\left(-d_{2, n}\right)-S_{t}^{n} \mathrm{e}^{\left(r(n-1)+\frac{1}{2} n(n-1) \sigma^{2}\right)(T-t)} N\left(-d_{1, n}\right)
$$

with

$$
\begin{gathered}
d_{1, n}=\frac{\ln \left(\frac{S_{t}^{n}}{K}\right)+n\left(r+\left(n-\frac{1}{2}\right) \sigma^{2}\right)(T-t)}{n \sigma \sqrt{T-t}} \\
d_{2, n}=\frac{\ln \left(\frac{S_{t}^{n}}{K}\right)+n\left(r-\frac{\sigma^{2}}{2}\right)(T-t)}{n \sigma \sqrt{T-t}}
\end{gathered}
$$

and

$$
P_{1}^{n}\left(S_{t}^{n}, t\right)=\frac{r K}{2 \pi i} \int_{c-i \infty}^{c+i \infty}\left(S_{t}^{n}\right)^{-\omega} \int_{t}^{T} \frac{\left(\hat{S}_{y}^{n}\right)^{\omega}}{\omega} \mathrm{e}^{\frac{1}{2} n^{2} \sigma^{2}\left(\omega^{2}+\alpha_{1} \omega-\alpha_{2}\right)(y-t)} \mathrm{d} y \mathrm{~d} \omega
$$

respectively. For (90) to hold as $T \rightarrow \infty$, it is necessary that $\operatorname{Re}\left(\omega^{2}+\alpha_{1} \omega-\alpha_{2}\right)<0$ i.e. $0<\operatorname{Re}(\omega)<\alpha_{2}$, where $\alpha_{2}$ is given by (89). The second smooth pasting condition (10) for a perpetual power put can be written as

$$
\frac{\partial E_{p}^{n}\left(\hat{S}_{\infty}^{n}, t\right)}{\partial \hat{S}_{\infty}^{n}}+\frac{\partial P_{1}^{n}\left(\hat{S}_{\infty}^{n}, t\right)}{\partial \hat{S}_{\infty}^{n}}=-1 \text { as } T \rightarrow \infty
$$

Differentiating (91) at $S_{t}^{n}=\hat{S}_{\infty}^{n}$ we have that

$$
\frac{\partial E_{p}^{n}\left(\hat{S}_{\infty}^{n}, t\right)}{\partial \hat{S}_{\infty}^{n}}=-\mathrm{e}^{\left(r(n-1)+\frac{1}{2} n(n-1) \sigma^{2}\right)(T-t)} N\left(-\hat{d}_{1, n}\right)
$$

where

$$
\hat{d}_{1, n}=\frac{\ln \left(\frac{\hat{S}_{\infty}^{n}}{K}\right)+n\left(r+\left(n-\frac{1}{2}\right) \sigma^{2}\right)(T-t)}{n \sigma \sqrt{T-t}}
$$


As $T \rightarrow \infty, \hat{d}_{1, n} \rightarrow \infty$ and therefore

$$
\frac{\partial E_{p}^{n}\left(\hat{S}_{\infty}^{n}, t\right)}{\partial \hat{S}_{\infty}^{n}} \rightarrow 0
$$

Also differentiating (92) yields

$$
\frac{\partial P_{1}^{n}\left(S_{t}^{n}, t\right)}{\partial S_{t}^{n}}=-\frac{r K}{2 \pi i} \int_{c-i \infty}^{c+i \infty}\left(S_{t}^{n}\right)^{-1}\left(\int_{t}^{T}\left(\frac{S_{t}^{n}}{\left(\hat{S}_{y}^{n}\right)}\right)^{-\omega} \mathrm{e}^{\frac{1}{2} n^{2} \sigma^{2}\left(\omega^{2}+\alpha_{1} \omega-\alpha_{2}\right)(y-t)} \mathrm{d} y\right) \mathrm{d} \omega
$$

Taking the limit of (97) as $T \rightarrow \infty$, we have

$$
\frac{\partial P_{1}^{n}\left(S_{t}^{n}, t\right)}{\partial S_{t}^{n}}=-\frac{r K}{2 \pi i} \int_{c-i \infty}^{c+i \infty}\left(S_{t}^{n}\right)^{-1}\left(\int_{t}^{\infty}\left(\frac{S_{t}^{n}}{\hat{S}_{\infty}^{n}}\right)^{-\omega} \mathrm{e}^{\frac{1}{2} n^{2} \sigma^{2}\left(\omega^{2}+\alpha_{1} \omega-\alpha_{2}\right)(y-t)} \mathrm{d} y\right) \mathrm{d} \omega
$$

Therefore,

$$
\frac{\partial P_{1}^{n}\left(S_{\infty}^{n}, t\right)}{\partial S_{\infty}^{n}}=\frac{K}{2 \pi i} \frac{2 r}{n^{2} \sigma^{2}} \int_{c-i \infty}^{c+i \infty} \frac{1}{\hat{S}_{\infty}^{n}\left(\omega^{2}+\alpha_{1} \omega-\alpha_{2}\right)} \mathrm{d} \omega
$$

where $\omega^{2}+\alpha_{1} \omega-\alpha_{2}=\left(\omega-\omega_{1}\right)\left(\omega-\omega_{2}\right)$ with $\omega \leq-1<0<\omega_{2} \leq \alpha_{2}$. The limiting cases $\omega_{1}=\alpha_{2}$ and $\omega_{2}=-1$ are the roots of $\omega^{2}+\alpha_{1} \omega-\alpha_{2}$. Hence (99) becomes

$$
\frac{\partial P_{1}^{n}\left(\hat{S}_{\infty}^{n}, t\right)}{\partial \hat{S}_{\infty}^{n}}=\frac{K}{2 \pi i} \frac{2 r}{n^{2} \sigma^{2}} \int_{c-i \infty}^{c+i \infty} \frac{1}{\hat{S}_{\infty}^{n}(\omega+1)\left(\omega-\alpha_{2}\right)} \mathrm{d} \omega
$$

Since $0<\operatorname{Re}(\omega)<\alpha_{2}$, application of the residue theorem given by (68) leads to

$$
\frac{\partial P_{1}^{n}\left(\hat{S}_{\infty}^{n}, t\right)}{\partial \hat{S}_{\infty}^{n}}=\alpha_{2} \frac{K}{\hat{S}_{\infty}^{n}\left(\omega_{2}-\omega_{1}\right)}=-\alpha_{2} \frac{K}{\hat{S}_{\infty}^{n}\left(\alpha_{2}+1\right)}
$$

Substituting (96) and (101) into (93) yields

$$
\begin{gathered}
0-\alpha_{2} \frac{K}{\hat{S}_{\infty}^{n}\left(\alpha_{2}+1\right)}=-1 \\
\hat{S}_{\infty}^{n}=K \frac{\alpha_{2}}{\left(\alpha_{2}+1\right)}
\end{gathered}
$$

Equation (102) is the expression for the free boundary of a perpetual American power put option. Next, we use (102) to derive an expression for the price of perpetual American power put option $A_{s}^{n}\left(S_{t}^{n}, t\right)$. Note that the price of a perpetual European power put option is zero, since it can never be exercised. Therefore, taking the limit as $T \rightarrow \infty$ in (90), the price of perpetual American put option for $S_{t}^{n}>\hat{S}_{\infty}^{n}$ is given by

$$
A_{\infty}\left(S_{t}^{n}, t\right)=\frac{r K}{2 \pi i} \int_{c-i \infty}^{c+i \infty}\left(\frac{S_{t}^{n}}{\hat{S}_{\infty}}\right)^{-\omega} \frac{1}{\omega}\left(\int_{t}^{\infty} \mathrm{e}^{\frac{1}{2} n^{2} \sigma^{2}\left(\omega^{2}+\alpha_{1} \omega-\alpha_{2}\right)(y-t)} \mathrm{d} y\right) \mathrm{d} \omega
$$

where $\operatorname{Re}\left(\omega^{2}+\alpha_{1} \omega-\alpha_{2}\right)<0$. Integrate the inner integral, (102) becomes

$$
A_{\infty}\left(S_{t}^{n}, t\right)=-\frac{r K}{2 \pi i} \frac{2}{n^{2} \sigma^{2}} \int_{c-i \infty}^{c+\infty}\left(\frac{S_{t}^{n}}{\hat{S}_{\infty}}\right)^{-\omega} \frac{1}{\omega(\omega+1)\left(\omega-\alpha_{2}\right)} \mathrm{d} \omega
$$

Once again we apply the residue theorem (68) to get 


$$
A_{\infty}^{n}\left(S_{t}^{n}, t\right)=\left(K-\hat{S}_{\infty}^{n}\right)\left(\frac{S_{t}^{n}}{\hat{S}_{\infty}^{n}}\right)^{-\alpha_{2}} \text { for } \hat{S}_{\infty}^{n}<S_{t}^{n}
$$

Equation (105) is the price of a perpetual American power put option obtained as a limit of the price of a finite-lived American power put option.

Theorem 11 (Dividend Yield) If $T \rightarrow \infty$ and $0<\operatorname{Re}(\omega)<\alpha_{1}^{*}$, then the free boundary of the perpetual American power put option is given by

$$
\bar{S}_{\infty}^{n}=\bar{S}_{\infty}^{n}(t)=K \frac{\omega_{1}}{\left(\omega_{1}+1\right)}
$$

and the price of perpetual American power put option equals

$$
A_{\infty}^{n}\left(S_{t}, t\right)=\left(K-\bar{S}_{\infty}^{n}\right)\left(\frac{S_{t}^{n}}{\bar{S}_{\infty}^{n}}\right)^{-\omega_{1}} \text { for } \bar{S}_{\infty}^{n}<S_{t}^{n}
$$

where

$$
\omega_{1}=\frac{-\alpha_{1}^{*}+\sqrt{\left(\alpha_{1}^{*}\right)^{2}+4 \alpha_{2}}}{2}
$$

and

$$
\alpha_{1}^{*}=\left(1-\frac{n-1}{n}-\frac{2(r-q)}{n \sigma^{2}}\right), \alpha_{2}=\frac{2 r}{n^{2} \sigma^{2}}
$$

Proof. The integral representation for the price of the American power put option which pays dividend yield given by (32) can be expressed as

$$
A_{p}^{n}\left(S_{t}^{n}, t\right)=E_{p}^{n}\left(S_{t}^{n}, t\right)+P_{1}^{n}\left(S_{t}^{n}, t\right)+P_{2}^{n}\left(S_{t}^{n}, t\right)
$$

where $E_{p}^{n}\left(S_{t}^{n}, t\right), P_{2}^{n}\left(S_{t}^{n}, t\right)$ and $P_{2}^{n}\left(S_{t}^{n}, t\right)$ are given by

$$
E_{p}^{n}\left(S_{t}^{n}, t\right)=K \mathrm{e}^{-r(T-t)} N\left(-d_{2, n}\right)-S_{t}^{n} \mathrm{e}^{\left(r(n-1)-n q+\frac{1}{2} n(n-1) \sigma^{2}\right)(T-t)} N\left(-d_{1, n}\right)
$$

with

$$
\begin{gathered}
d_{1, n}=\frac{\ln \left(\frac{S_{t}^{n}}{K}\right)+n\left(r-q+\left(n-\frac{1}{2}\right) \sigma^{2}\right)(T-t)}{n \sigma \sqrt{T-t}} \\
d_{2, n}=\frac{\ln \left(\frac{S_{t}^{n}}{K}\right)+n\left(r-q-\frac{\sigma^{2}}{2}\right)(T-t)}{n \sigma \sqrt{T-t}} \\
P_{1}^{n}\left(S_{t}^{n}, t\right)=\frac{r K}{2 \pi i} \int_{c-i \infty}^{c+i \infty}\left(S_{t}^{n}\right)^{-\omega} \int_{t}^{T} \frac{\left(\bar{S}_{y}^{n}\right)^{\omega}}{\omega} \mathrm{e}^{\frac{1}{2} n^{2} \sigma^{2}\left(\omega^{2}+\alpha_{1}^{*} \omega-\alpha_{2}\right)(y-t)} \mathrm{d} y \mathrm{~d} \omega \\
P_{2}^{n}\left(S_{t}^{n}, t\right)=-\frac{q}{2 \pi i} \int_{c-i \infty}^{c+i \infty}\left(S_{t}^{n}\right)^{-\omega} \int_{t}^{T} \frac{\left(\bar{S}_{y}^{n}\right)^{\omega+1}}{\omega+1} \mathrm{e}^{\frac{1}{2} n^{2} \sigma^{2}\left(\omega^{2}+\alpha_{1}^{*} \omega-\alpha_{2}\right)(y-t)} \mathrm{d} y \mathrm{~d} \omega
\end{gathered}
$$

respectively. The roots of $\omega^{2}+\alpha_{1}^{*} \omega-\alpha_{2}$ are

$$
\omega_{1}=\frac{-\alpha_{1}^{*}+\sqrt{\left(\alpha_{1}^{*}\right)^{2}+4 \alpha_{2}}}{2}
$$


and

$$
\omega_{2}=\frac{-\alpha_{1}^{*}-\sqrt{\left(\alpha_{1}^{*}\right)^{2}+4 \alpha_{2}}}{2}
$$

Thus we write that

$$
\omega^{2}+\alpha_{1}^{*} \omega-\alpha_{2}=\left(\omega-\omega_{1}\right)\left(\omega-\omega_{2}\right)
$$

with $\omega \leq-1<0<\omega_{2} \leq \alpha_{2}$. For (110) to hold as $T \rightarrow \infty$, it is necessary that $\operatorname{Re}\left(\omega^{2}+\alpha_{1}^{*} \omega-\alpha_{2}\right)<0$ i.e. $0<\operatorname{Re}(\omega)<\alpha_{2}$, where $\alpha_{1}^{*}$ and $\alpha_{2}$ are given by (109). The second smooth pasting condition (10) for a perpetual power put which pays dividend yield can be written as

$$
\frac{\partial E_{p}^{n}\left(\bar{S}_{\infty}^{n}, t\right)}{\partial \bar{S}_{\infty}^{n}}+\frac{\partial P_{1}^{n}\left(\bar{S}_{\infty}^{n}, t\right)}{\partial \bar{S}_{\infty}^{n}}+\frac{\partial P_{2}^{n}\left(\bar{S}_{\infty}^{n}, t\right)}{\partial \bar{S}_{\infty}^{n}}=-1 \text { as } T \rightarrow \infty
$$

Differentiating (111) at $S_{t}^{n}=\bar{S}_{\infty}^{n}$ we have that

$$
\frac{\partial E_{p}^{n}\left(\bar{S}_{\infty}^{n}, t\right)}{\partial \bar{S}_{\infty}^{n}}=-\mathrm{e}^{\left(r(n-1)-n q+\frac{1}{2} n(n-1) \sigma^{2}\right)(T-t)} N\left(-\bar{d}_{1, n}\right)
$$

where

$$
\bar{d}_{1, n}=\frac{\ln \left(\frac{\bar{S}_{\infty}^{n}}{K}\right)+n\left(r-q+\left(n-\frac{1}{2}\right) \sigma^{2}\right)(T-t)}{n \sigma \sqrt{T-t}}
$$

As $T \rightarrow \infty, \bar{d}_{1, n} \rightarrow \infty$ and therefore

$$
\frac{\partial E_{p}^{n}\left(\bar{S}_{\infty}^{n}, t\right)}{\partial \bar{S}_{\infty}^{n}} \rightarrow 0
$$

Now differentiating (112) w.r.t $S_{t}^{n}$ and taking the limit $T \rightarrow \infty$ we have that

$$
\frac{\partial P_{1}^{n}\left(S_{t}^{n}, t\right)}{\partial S_{t}^{n}}=-\frac{r K}{2 \pi i} \int_{c-i \infty}^{c+i \infty}\left(S_{t}^{n}\right)^{-1}\left(\int_{t}^{\infty}\left(\frac{S_{t}^{n}}{\bar{S}_{\infty}^{n}}\right)^{-\omega} \mathrm{e}^{\frac{1}{2} n^{2} \sigma^{2}\left(\omega^{2}+\alpha_{1}^{*} \omega-\alpha_{2}\right)(y-t)} \mathrm{d} y\right) \mathrm{d} \omega
$$

Therefore, by setting $S_{t}^{n}=\bar{S}_{\infty}^{n}$ we have that

$$
\begin{gathered}
\frac{\partial P_{1}^{n}\left(\bar{S}_{\infty}^{n}, t\right)}{\partial \bar{S}_{\infty}^{n}}=\frac{K}{2 \pi i} \frac{2 r}{n^{2} \sigma^{2}} \int_{c-i \infty}^{c+i \infty} \frac{1}{\bar{S}_{\infty}^{n}\left(\omega^{2}+\alpha_{1}^{*} \omega-\alpha_{2}\right)} \mathrm{d} \omega \\
\text { Let } \frac{2 r}{n^{2} \sigma^{2}}=\alpha_{2} \text { and } \omega^{2}+\alpha_{1}^{*} \omega-\alpha_{2}=\left(\omega-\omega_{1}\right)\left(\omega-\omega_{2}\right) \\
\quad \frac{\partial P_{1}^{n}\left(\bar{S}_{\infty}^{n}, t\right)}{\partial \bar{S}_{\infty}^{n}}=\frac{K}{2 \pi i} \alpha_{2} \int_{c-i \infty}^{c+i \infty} \frac{1}{\bar{S}_{\infty}^{n}\left(\omega-\omega_{1}\right)\left(\omega-\omega_{2}\right)} \mathrm{d} \omega
\end{gathered}
$$

In the same manner, setting $T \rightarrow \infty$ and differentiating (113) w.r.t $S_{t}^{n}$, we have that

$$
\frac{\partial P_{2}^{n}\left(\bar{S}_{t}^{n}, t\right)}{\partial \bar{S}_{t}^{n}}=\frac{q}{2 \pi i} \int_{c-i \infty}^{c+i \infty}\left(\int_{t}^{\infty} \frac{\omega}{\omega+1}\left(\frac{S_{t}^{n}}{\bar{S}_{\infty}^{n}}\right)^{-(\omega+1)} \mathrm{e}^{\frac{1}{2} n^{2} \sigma^{2}\left(\omega^{2}+\alpha_{1}^{*} \omega-\alpha_{2}\right)(y-t)} \mathrm{d} y\right) \mathrm{d} \omega
$$

Therefore, 


$$
\begin{aligned}
\frac{\partial P_{2}^{n}\left(\bar{S}_{t}^{n}, t\right)}{\partial \bar{S}_{t}^{n}}= & \frac{1}{2 \pi i}\left(\frac{\sigma^{2} n(n-1)+2 n(r-q)-2 r}{n^{2} \sigma^{2}}\right) \\
& \times \int_{c-i \infty \infty}^{c+i \infty} \frac{\omega}{\omega+1}\left(\frac{S_{t}^{n}}{\bar{S}_{\infty}^{n}}\right)^{-(\omega+1)} \frac{1}{\left(\omega^{2}+\alpha_{1}^{*} \omega-\alpha_{2}\right)} \mathrm{d} \omega
\end{aligned}
$$

Setting $S_{t}^{n}=S_{\infty}^{n}$ and solving (121) further we have that

$$
\frac{\partial P_{2}^{n}\left(\bar{S}_{\infty}^{n}, t\right)}{\partial \bar{S}_{\infty}^{n}}=\frac{1}{2 \pi i}\left(\frac{\sigma^{2} n(n-1)+2 n(r-q)-2 r}{n^{2} \sigma^{2}}\right) \int_{c-i \infty}^{c+i \infty} \frac{\omega}{(\omega+1)\left(\omega-\omega_{1}\right)\left(\omega-\omega_{2}\right)} \mathrm{d} \omega
$$

Once again by the application of residue theorem (68), then (120) and (122) yield

$$
\frac{\partial P_{1}^{n}\left(\bar{S}_{\infty}^{n}, t\right)}{\partial \bar{S}_{\infty}^{n}}=\alpha_{2} K \frac{1}{\bar{S}_{\infty}^{n}\left(\omega_{1}-\omega_{2}\right)}=-\alpha_{2} \frac{K}{\bar{S}_{\infty}^{n}\left(\alpha_{2}+1\right)}
$$

and

$$
\frac{\partial P_{2}^{n}\left(\bar{S}_{\infty}^{n}, t\right)}{\partial \bar{S}_{\infty}^{n}}=\left(1-\alpha_{1}^{*}-\alpha_{2}\right)\left(\frac{\omega_{2}}{\left(\omega_{2}+1\right)\left(\omega_{2}-\omega_{1}\right)}-\frac{1}{\left(\omega_{1}+1\right)\left(\omega_{2}+1\right)}\right)
$$

respectively. Substituting (117), (123) and (124) into (114), we obtain

$$
\bar{S}_{\infty}^{n}=\frac{\omega_{1}}{\left(\omega_{1}+1\right)} K
$$

Equation (125) is called the free boundary of the perpetual American power put option which pays dividend yield.

The price for the perpetual American power put option is given by

$$
\begin{aligned}
A_{\infty}^{n}\left(S_{t}^{n}, t\right)= & -\frac{\alpha_{2} K}{2 \pi i} \int_{c-i \infty}^{c+\infty}\left(\frac{S_{t}^{n}}{\bar{S}_{\infty}^{n}}\right)^{-\omega} \frac{1}{\omega\left(\omega-\omega_{1}\right)\left(\omega-\omega_{2}\right)} \mathrm{d} \omega \\
& -\frac{1}{2 \pi i}\left(\frac{\sigma^{2} n(n-1)+2 n(r-q)-2 r}{n^{2} \sigma^{2}}\right) \\
& \times \int_{c-i \infty}^{c+\infty} \bar{S}_{\infty}^{n}\left(\frac{S_{t}^{n}}{\bar{S}_{\infty}^{n}}\right)^{-\omega} \frac{1}{(\omega+1)\left(\omega-\omega_{1}\right)\left(\omega-\omega_{2}\right)} \mathrm{d} \omega
\end{aligned}
$$

Using the residue theorem (68), then (126) becomes

$$
\begin{aligned}
A_{\infty}^{n}\left(S_{t}^{n}, t\right)= & \left(\frac{S_{t}^{n}}{\bar{S}_{\infty}^{n}}\right)^{-\omega_{1}} \frac{\alpha_{2} K}{\omega_{1}\left(\omega_{1}-\omega_{2}\right)}+\left(\frac{\sigma^{2} n(n-1)+2 n(r-q)-2 r}{n^{2} \sigma^{2}}\right) \\
& \times\left(\frac{S_{t}^{n}}{\bar{S}_{\infty}^{n}}\right) \frac{\bar{S}_{\infty}^{n}}{\left(\omega_{1}+1\right)\left(\omega_{1}-\omega_{2}\right)} \\
= & \left(K-\bar{S}_{\infty}^{n}\right)\left(\frac{S_{t}^{n}}{\bar{S}_{\infty}^{n}}\right)^{-\omega_{1}}
\end{aligned}
$$

\section{Numerical Experiments}

In this section we present some numerical experiments and discussion of results.

\section{Experiment 1}

We consider the valuation of the American power put option for $n=\{1.90,1.95,2.00,2.05,2.10\}$ which pays dividend yield $q=0.06$ with the following parameters: 


$$
S_{t}=\$ 10, K=\$ 100, \sigma=\{0.1,0.15,0.20,0.25,0.30\}, r=0.08, T=0.5
$$

The result generated is shown in Table 1 below.

\section{Experiment 2}

We consider the valuation of the American power put option for $n=1$ which pays non-dividend yield with the following parameters:

$$
S_{t}=\$ 40, K=\{\$ 35, \$ 40, \$ 45\}, T=0.083, r=0.05, \sigma=\{0.2,0.3,0.4\}
$$

The results generated for the price of the American power put option via Black-Scholes model (BSM), binomial model (BM) and the Mellin transform method (MTM) are shown in Tables 2-4 below. Also the results generated for the free boundary of the American power put option are shown in Tables 5-7 below.

\section{Experiment 3}

We consider the valuation of the American Power put option with the following parameters:

$$
S_{t}=\$ 40, K=\{\$ 35, \$ 40, \$ 45\}, T=0.583, r=0.05, \sigma=0.4, n=1, c=2
$$

The comparative results analysis of the Mellin transform method (MTM) in the context of Black-Scholes model (BSM), binomial model (BM), recursive method (RM) and Finite difference method (FDM) are shown in

\begin{tabular}{|c|c|c|c|c|c|}
\hline$\sigma \backslash n$ & 1.90 & 1.95 & 2.0 & 2.05 & 2.10 \\
\hline 0.1 & 18.274 & 10.289 & 4.354 & 1.309 & 0.275 \\
\hline 0.15 & 18.997 & 12.147 & 6.809 & 3.316 & 1.403 \\
\hline 0.20 & 20.160 & 14.102 & 9.175 & 5.548 & 3.125 \\
\hline 0.25 & 21.535 & 16.058 & 11.453 & 7.832 & 5.129 \\
\hline 0.30 & 23.008 & 17.981 & 13.653 & 10.077 & 7.251 \\
\hline
\end{tabular}
Table 8.

Table 1. American power put values.

Table 2. The price of American power put option using $n=1, c=2, \sigma=0.2, r=0.05, S_{t}=\$ 40, T=0.083$.

\begin{tabular}{cccc}
\hline$K$ & Black-Scholes Model (BSM) & Binomial Model (BM) & Mellin Transform Method (MTM) \\
\hline 35 & 0.006 & 0.006 & 0.007 \\
40 & 0.840 & 0.851 & 0.852 \\
45 & 4.840 & 5.000 & 5.031 \\
\hline
\end{tabular}

Table 3. The price of American power put option using $n=1, c=2, \sigma=0.3, r=0.05, S_{t}=\$ 40, T=0.083$.

\begin{tabular}{cccc}
\hline$K$ & Black-Scholes Model (BSM) & Binomial Model (BM) & Mellin Transform Method (MTM) \\
\hline 35 & 0.076 & 0.076 & 0.078 \\
40 & 1.295 & 1.310 & 1.310 \\
45 & 4.975 & 5.051 & 5.058 \\
\hline
\end{tabular}

Table 4. The price of American power put option using $n=1, c=2, \sigma=0.4, r=0.05, S_{t}=\$ 40, T=0.083$.

\begin{tabular}{cccc}
\hline$K$ & Black-Scholes Model (BSM) & Binomial Model (BM) & Mellin Transform Method (MTM) \\
35 & 0.244 & 0.245 & 0.247 \\
40 & 1.753 & 1.766 & 1.768 \\
45 & 5.231 & 5.285 & 5.300 \\
\hline
\end{tabular}


Table 5. The free boundary of American power put option using $n=1, c=2, \sigma=0.2, r=0.05, T=0.083$.

\begin{tabular}{ccc}
\hline Strike Price, $K$ & Underlying Asset Price, $S_{t}$ & Free Boundary $\hat{S}_{t}$ \\
\hline 35 & 40 & 31.740 \\
40 & 40 & 36.273 \\
45 & 40 & 40.810 \\
\hline
\end{tabular}

Table 6. The free boundary of American power put option using $n=1, c=2, \sigma=0.3, r=0.05, T=0.083$.

\begin{tabular}{ccc}
\hline Strike Price, $K$ & Underlying Asset Price, $S_{t}$ & Free Boundary $\hat{S}_{t}$ \\
\hline 35 & 40 & 29.783 \\
40 & 40 & 34.040 \\
45 & 40 & 38.291 \\
\hline
\end{tabular}

Table 7. The free boundary of American power put option using $n=1, c=2, \sigma=0.4, r=0.05, T=0.083$.

\begin{tabular}{ccc}
\hline Strike Price, $K$ & Underlying Asset Price, $S_{t}$ & Free Boundary $\hat{S}_{t}$ \\
\hline 35 & 40 & 27.850 \\
40 & 40 & 31.830 \\
45 & 40 & 35.804 \\
\hline
\end{tabular}

Table 8. The comparative results analysis of some numerical methods for the valuation of American power put option.

\begin{tabular}{cccccccccccc}
\hline$K$ & $S_{t}$ & $\sigma$ & $c$ & $n$ & $r$ & $T$ & BSM & BM & MTM & RM & FDM \\
\hline 35 & 40 & 0.4 & 2 & 1 & 0.05 & 0.083 & 2.104 & 2.144 & 2.157 & 2.160 & 2.168 \\
40 & 40 & 0.4 & 2 & 1 & 0.05 & 0.083 & 4.232 & 4.330 & 4.354 & 4.370 & 4.357 \\
45 & 40 & 0.4 & 2 & 1 & 0.05 & 0.083 & 7.144 & 7.364 & 7.384 & 7.390 & 7.380 \\
\hline
\end{tabular}

\section{Discussion of Results}

From Figure 1 below, we observe that the higher the volatility, the higher the values of the American power put option. Also the higher the power of the American put option, the lower the values of the option. Figures 2-4 below show that the Mellin transform method is mutually consistent, performs very well, accurate and agrees with the values of Black-Scholes model (BSM). In Figure 5 below, we plot the free boundary $\hat{S}_{t}$ as a function of the strike price $K$ for different values of volatility $\sigma$. We observe that the higher the volatility, the lower the optimal exercise boundary of the American power put option. Figure 6 below demonstrates that the Mellin transform method is a better alternative technique compared to the Black-Scholes model (BSM), binomial model $(B M)$, recursive method $(\mathrm{RM})$ and finite difference method $(\mathrm{BM})$ for the valuation of the American power put option. Hence the Mellin transform method is a good technique for the valuation of the American power put option.

\section{Conclusion}

In this paper, we have derived the integral representations for the price and the free boundary of the American power put option for non-dividend and dividend yields using the Mellin transform method. We also extended the integral equation for the price of the American power put option to derive the expression for the free boundary and the price of the perpetual American power put option which pays both non-dividend and dividend yields as the limit of a finite-lived option by means of smooth pasting condition. In general, numerical experiments have shown that the Mellin transform method is accurate, flexible, efficient and produces accurate prices for the optimal exercise boundary for a wide range of parameters. 


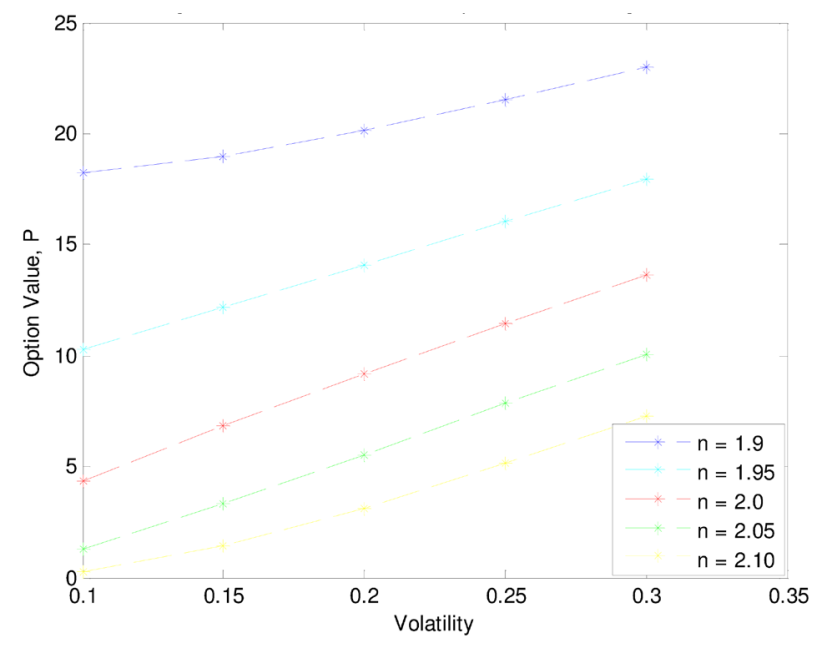

Figure 1. American power put option values using Table 1.

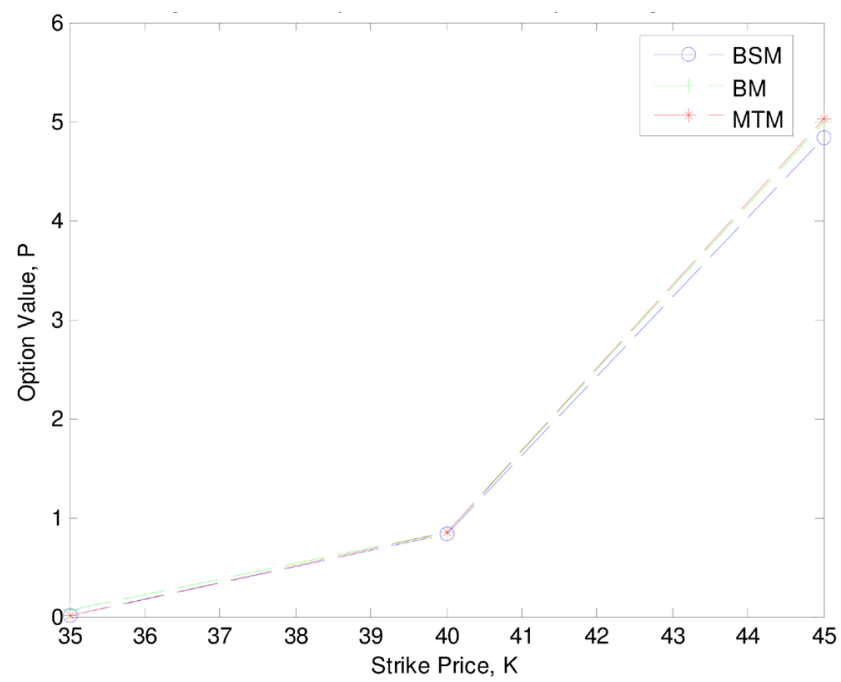

Figure 2. The comparative results analysis using Table 2.

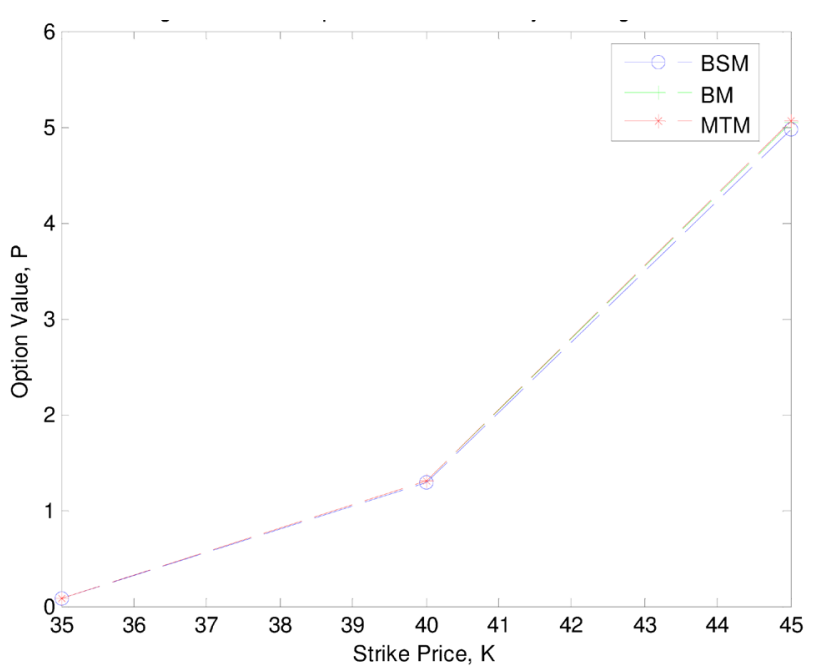

Figure 3. The comparative results analysis using Table 3. 


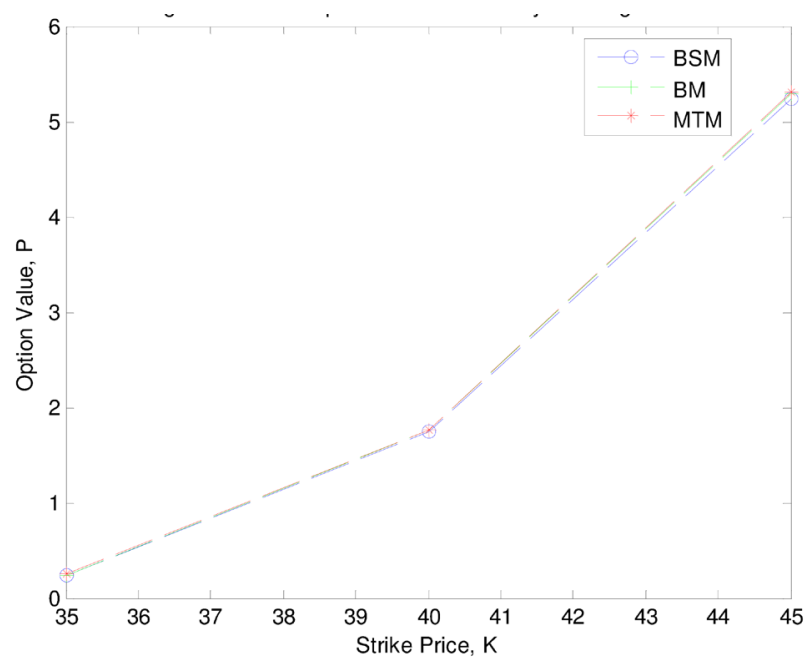

Figure 4. The comparative results analysis using Table 4.

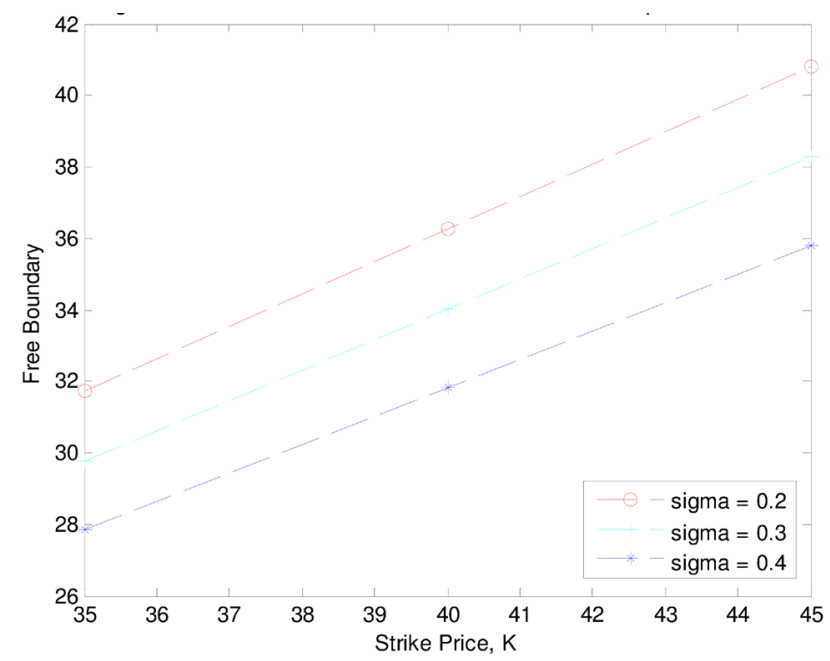

Figure 5. The free boundaries of American power put option with $\mathrm{n}=1$.

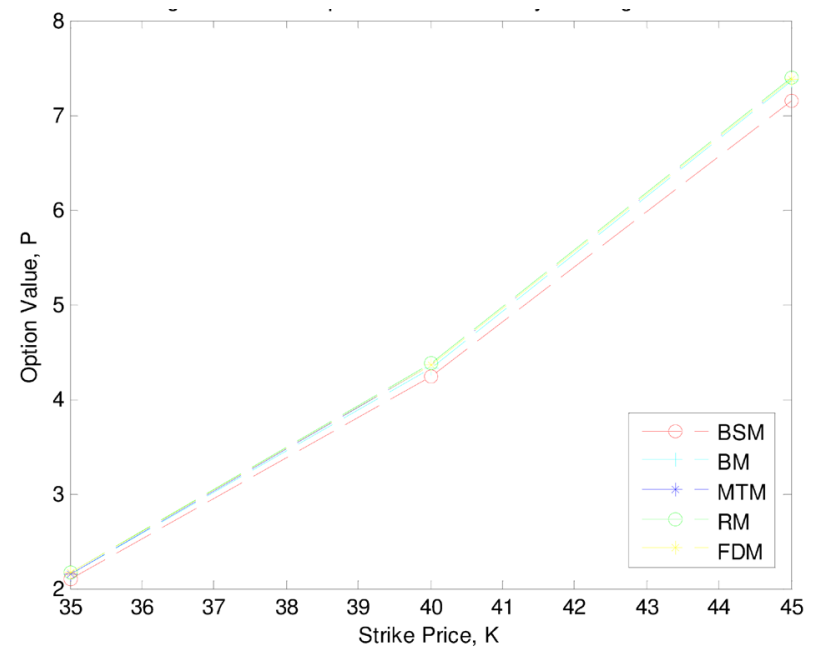

Figure 6. The comparative results analysis using Table 8. 


\section{References}

[1] Samuelson, P.A. (1965) Rational Theory of Warrant Pricing. Industrial Management Review, 6, 13-31.

[2] Panini, R. and Srivastav, R.P. (2005) Pricing Perpetual Options Using Mellin Transforms. Applied Mathematics Letters, 18, 471-474. http://dx.doi.org/10.1016/j.aml.2004.03.012

[3] Frontczak, R. and Schöbel, R. (2008) Pricing American Options with Mellin Transforms. Working Paper.

[4] Nwozo, C.R. and Fadugba, S.E. (2014) Mellin Transform Method for the Valuation of Some Vanilla Power Options with Non-Dividend Yield. International Journal of Pure and Applied Mathematics, 96, 79-104. http://dx.doi.org/10.12732/ijpam.v96i1.7

[5] Kim, I. (1990) The Analytic Valuation of American Options. The Review of Financial Studies, 3, 547-572. http://dx.doi.org/10.1093/rfs/3.4.547

[6] Kim, I. and Yu, G.G. (1996) An Alternative Approach to the Valuation of American Options and Applications. Review of Derivatives Research, 1, 61-85. http://dx.doi.org/10.1007/BF01536395

[7] Erdelyi, A., Magnus, W., Oberhettinger, F. and Tricomi, F. (1954) Tables of Integral Transforms, Vol. 1-2. McGrawHill, New York.

[8] Fadugba, S.E. and Nwozo, C.R. (2015) Integral Representations for the Price of Vanilla Put Options on a Basket of Two-Dividend Paying Stocks. Applied Mathematics, 6, 783-792. http://dx.doi.org/10.4236/am.2015.65074

[9] Frontczak, R. and Schöbel, R. (2009) On Modified Mellin Transforms, Gauss-Laguerre Quadrature and the Valuation of American Call Options. Tübinger Diskussionsbeiträge, No. 320.

[10] Gradshteyn, I. and Ryshik, I. (2007) Table of Integrals Series and Products. 7th Edition, Academic Press, Waltham.

[11] Panini, R. and Srivastav, R.P. (2004) Option Pricing with Mellin Transforms. Mathematical and Computer Modelling, 40, 43-56. http://dx.doi.org/10.1016/j.mcm.2004.07.008

[12] Vasilieva, O. (2009) A New Method of Pricing Multi-Options Using Mellin Transforms and Integral Equations. Master's Thesis in Financial Mathematics, School of Information Science, Computer and Electrical Engineering, Halmstad University, Halmstad.

[13] Nguyen, T.H. and Yakubovich, S.B. (1991) The Double Mellin-Barnes Type Integrals and Their Applications to Convolution Theory. World Scientific, Series on Soviet Mathematics, Vol. 6, Singapore, 295p.

[14] Zemanian, A.H. (1987) Generalized Integral Transformations. Dover Publications, New York.

[15] Zieneb, A.E. and Rokiah, R.A. (2011) Analytical Solution for an Arithmetic Asian Option Using Mellin Transforms. International Journal of Mathematical Analysis, 5, 1259-1265. 